\title{
WORD MAPS, WORD MAPS WITH CONSTANTS AND REPRESENTATION VARIETIES OF ONE-RELATOR GROUPS
}

\author{
NIKOLAI GORDEEV, BORIS KUNYAVSKIĬ, EUGENE PLOTKIN
}

To Efim Zelmanov on the occasion of his 60th birthday

\begin{abstract}
We consider word maps and word maps with constants on a simple algebraic group $G$. We present results on the images of such maps, in particular, we prove a theorem on the dominance of general word maps with constants, which can be viewed as an analogue of a well-known theorem of Borel on the dominance of genuine word maps. Besides, we establish a relationship between the existence of unipotents in the image of the map induced by $w \in F_{m}$ and the structure of the representation variety $R\left(\Gamma_{w}, G\right)$ of the group $\Gamma_{w}=F_{m} /\langle w\rangle$.
\end{abstract}

\section{INTRODUCTION}

Word maps. Let $F_{m}$ be the free group of rank $m$. Fix its generators $x_{1}, \ldots, x_{m}$. Then for any word $w=w\left(x_{1}, \ldots, x_{m}\right) \in F_{m}$ and any group $G$ one can define the word map

$$
\widetilde{w}: G^{m} \rightarrow G
$$

by evaluation. Namely, $\widetilde{w}\left(g_{1}, \ldots, g_{m}\right)$ is obtained by substituting $g_{i}$ in place of $x_{i}$ and $g_{i}^{-1}$ in place of $x_{i}^{-1}$ followed by computing the resulting value $w\left(g_{1}, \ldots, g_{m}\right)$.

Word maps have been intensely studied over at least two past decades in various contexts (see, e.g., [Se], Shal], [BGK], [KBKP] for surveys). In this paper, we consider the case where $G=\mathcal{G}(K)$ is the group of $K$-points of a simple linear algebraic group $\mathcal{G}$ defined over an algebraically closed field $K$. We are mainly interested in studying the image of $\widetilde{w}$. Borel's theorem [Bo1] says that $\widetilde{w}$ is dominant, i.e., its image contains a Zariski dense open subset of $G$. However, $\widetilde{w}$ may not be surjective: this may happen in the case of power maps on groups with non-trivial centre (say, squaring map on $\operatorname{SL}(2, \mathbb{C})$ ) and, if $\mathcal{G}$ is not of type A, even on adjoint groups, see [Ch1], Ch2], [Stei]. For the adjoint groups of type A, the surjectivity problem is wide open, even in the case of groups of rank 1, and even for words in two variables.

The goal of the present paper is two-fold. First, we extend our viewpoint on the dominance and surjectivity problems from genuine word maps to word maps with constants and establish a partial, "generic" analogue of Borel's dominance theorem. Another extension concerns a continuation of the word map $\widetilde{w}: \mathrm{GL}_{n}(K)^{m} \rightarrow \mathrm{GL}_{n}(K)$ to the map $\widetilde{w}^{*}: \mathrm{M}_{n}(K)^{m} \rightarrow \mathrm{M}_{n}(K)$. Being interesting in its own right, this method yields, as a byproduct, a new proof of some results of Bandman and Zarhin [BZ], who proved the surjectivity of $\widetilde{w}$ for $G=\mathrm{PGL}_{2}(K)$ in the case where $K$ is an algebraically closed field of characteristic zero, $m=2$, and $w \in F_{m} \backslash F_{m}^{2}$, where $F_{m}^{1}=\left[F_{m}, F_{m}\right], \ldots, F_{m}^{i}=\left[F_{m}^{i-1}, F_{m}^{i-1}\right], \ldots$ Our second goal consists in studying the geometric structure of the representation variety of the one-relator group $\Gamma_{w}:=F_{m} /\langle w\rangle$ with an eye towards applying the data on its 
irreducible components to searching unipotent elements in the image of the word map. This often allows one to prove the surjectivity of the word map on $\mathrm{PGL}_{2}(K)$. We give a non-trivial example of such a $w \in F_{2}$, in the spirit of [BZ] but avoiding their computer calculations.

Word maps with constants. Let $G$ be a group, let

$$
\Sigma=\left\{\sigma_{1}, \ldots, \sigma_{r} \mid \sigma_{i} \in G \backslash Z(G) \text { for every } i=1, \ldots, r\right\},
$$

and let $w_{1}, \ldots, w_{r+1} \in F_{m}$ be reduced words. The expression

$$
w_{\Sigma}=w_{1} \sigma_{1} w_{2} \sigma_{2} \cdots w_{r} \sigma_{r} w_{r+1}
$$

is called a word with constants (or a generalized monomial) if the sequence $w_{2}, \ldots, w_{r}$ does not contain the identity word. We will view a word $w \in F_{m}$ as a word with constants $w_{\Sigma}$ with $\Sigma=\emptyset$ and $w=w_{1}$.

A word with constants also induces a map

$$
\widetilde{w}_{\Sigma}: G^{m} \rightarrow G
$$

Let now $G=\mathcal{G}(K)$ where $\mathcal{G}$ is a simple algebraic group defined over an algebraically closed field $K$. In general, the image $\operatorname{Im}_{\widetilde{w}_{\Sigma}}$ is not dense in $G$ as in Borel's Theorem. However, there are examples when this image is dense. For instance, the problem of the density of $\operatorname{Im}_{\widetilde{w}_{\Sigma}}$ is related to the definition of covering numbers for products of conjugacy classes (see [G1]). Namely, let $r=m$ and let

$$
w_{\Sigma}=x_{1} \sigma_{1} x_{1}^{-1} x_{2} \sigma_{2} x_{2}^{-1} \cdots x_{m} \sigma_{m} x_{m}^{-1} .
$$

Then $\operatorname{Im}_{\widetilde{w}_{\Sigma}}=C_{1} C_{2} \cdots C_{m}$ where $C_{i}$ is the conjugacy class of $\sigma_{i}$. Thus,

$$
\overline{\operatorname{Im}_{\widetilde{w}_{\Sigma}}}=\overline{C_{1} C_{2} \cdots C_{m}}
$$

where $\bar{X}$ is the Zariski closure of $X \subset G$. In [G1] it has been proved that $\overline{\operatorname{Im}_{\widetilde{w}_{\Sigma}}}=G$ if $|\Sigma|>2 \operatorname{rank} G+1$.

In the present paper, we prove (Theorem 1.4) that for a "general" word with constants $w_{\Sigma}$ the induced map $\widetilde{w}_{\Sigma}$ turns out to be dominant.

Note that words with constants are also related to other problems in the theory of algebraic groups (see, e.g., [G2], [KT], [Ste1], [Ste2]).

Word maps and representation varieties.

For a simple algebraic group $\mathcal{G}$ defined over an algebraically closed field $K$ one can define the quotient map $\pi: \mathcal{G} \rightarrow \mathcal{G} / / \mathcal{G} \approx T / W$ where $\mathcal{G} / / \mathcal{G}$ and $T / W$ are categorical quotients with respect to the action of $\mathcal{G}$ on $\mathcal{G}$ by conjugation and the natural action of the Weyl group $W$ on a maximal torus $T$, respectively, see [SS] for details. Then we have the map

$$
\pi \circ \widetilde{w}: \mathcal{G}^{m} \rightarrow T / W .
$$

We denote by $\widetilde{w}_{K}: \mathcal{G}(K)^{m} \rightarrow \mathcal{G}(K)$ the induced word map on the group $G:=\mathcal{G}(K)$. Borel's theorem implies that $\operatorname{Im}\left(\pi \circ \widetilde{w}_{K}\right)$ is dense in $T / W$. However, we do not know when $\operatorname{Im}\left(\pi \circ \widetilde{w}_{K}\right)=T(K) / W$. Moreover, we have no example when $\operatorname{Im}\left(\pi \circ \widetilde{w}_{K}\right) \neq T(K) / W$. On the other hand, we have not so many examples when $\operatorname{Im}\left(\pi \circ \widetilde{w}_{K}\right)=T(K) / W$. The latter equality holds, say, for the Engel words $w=[\cdots[[x, y], y], \cdots y]$ (see [G3]) and for the 
power words $w=x^{k}$. Bandman and Zarhin proved in [BZ] that $\operatorname{Im}\left(\pi \circ \widetilde{w}_{K}\right)=T(K) / W$ when $G=\mathrm{SL}_{2}(K)$.

Note that the equality $\operatorname{Im}\left(\pi \circ \widetilde{w}_{K}\right)=T(K) / W$ implies that for every semisimple element $s \in T(K)$ one can find an element of the form $s u$ in the image of $\widetilde{w}_{K}$ where $u$ is a unipotent element of $G$ which commutes with $s$, see [SS]. This implies, in its turn, that

$$
\operatorname{Im}\left(\pi \circ \widetilde{w}_{K}\right)=T(K) / W \Rightarrow \text { all regular semisimple elements of } G \text { belong to } \operatorname{Im} \widetilde{w}_{K} \text {. }
$$

Thus, if $\mathcal{G}=\mathrm{SL}_{2}$ then every element of $G$ belongs to $\operatorname{Im} \widetilde{w}_{K}$ except, possibly, $-1, \pm u$ where $u$ is a unipotent element. Hence, if $\mathcal{G}=\mathrm{PGL}_{2}$ then $\operatorname{Im} \widetilde{w}_{K} \supseteq G \backslash\{u\}$. Then we have only one obstacle to proving the surjectivity of $\widetilde{w}_{K}$ for $\mathrm{PGL}_{2}(K)$, namely, we have to prove the existence of a non-trivial unipotent element in the image. The existence of unipotent elements in $\operatorname{Im} \widetilde{w}_{K}$ is somehow related to the structure of the representation variety. Namely, denote

$$
\mathcal{W}_{w}=\widetilde{w}^{-1}(1), \mathcal{T}_{w}=(\pi \circ \widetilde{w})^{-1}(\pi(1))
$$

(here we denote by 1 the identity of $G$ ). Then $\mathcal{W}_{w}$ is the representation variety $R(\Gamma, G)$ where $\Gamma=\Gamma_{w}$ is the $m$-generated group with one defining relation $w$ (see, e.g., [LM]). The set $\mathcal{T}_{w} \subset G^{m}$ is the set of $m$-tuples $\left(g_{1}, \ldots, g_{m}\right) \in G^{m}$ such that

$$
w\left(g_{1}, \ldots, g_{n}\right)=u \text { is a unipotent element of } G,
$$

that is, $\mathcal{T}_{w}$ is the preimage of the unipotent subvariety of $G$. We have an inclusion $\mathcal{W}_{w} \subseteq \mathcal{T}_{w}$ of affine subsets of $G^{m}$, and the inequality $\mathcal{W}_{w} \neq \mathcal{T}_{w}$ is a sufficient condition for the existence of a non-trivial unipotent element in $\operatorname{Im} \widetilde{w}$. We calculate several examples of words in $F_{2}$ for the group $\mathcal{G}=\mathrm{SL}_{2}$. In all these examples $\mathcal{W}_{w} \neq \mathcal{T}_{w}$ but there are cases when some irreducible components of $\mathcal{W}_{w}$ coincide with a irreducible component of $\mathcal{T}_{w}$. Possibly, the investigation of structure properties of the representation varieties $\mathcal{W}_{w}$ would give an answer to the question on the existence a non-trivial unipotent element in $\mathrm{SL}_{2}(\mathbb{C})$ lying in the image of $\widetilde{w}$.

Some results of this paper were announced in GKP.

\section{Notation and conventions.}

Below, if not stated otherwise, $K$ is an algebraically closed field and $\mathcal{G}$ is an algebraic group defined over $K$, so we identify the group $\mathcal{G}$ with $G:=\mathcal{G}(K)$.

We denote the identity element of $G$ by 1 ;

$N_{G}(H)$ denotes the normalizer of $H$ in $G$;

$R * Q$ denotes the free product of groups $R$ and $Q$;

for a group $\Delta$ and $x, y \in \Delta$, we use the symbol $x \sim y$ if $x$ is conjugate to $y$ in $\Delta$;

$\mathbb{G}_{\mathrm{a}}, \mathrm{GL}_{n}, \mathrm{SL}_{n}$ denote the additive, general linear, special linear groups;

$\mathrm{M}_{n}(K)$ is the set of $n \times n$-matrices over $K$;

$I_{n} \in \mathrm{M}_{n}(K)$ is the identity matrix;

for $A \in \mathrm{M}_{n}(K)$ by $A^{*}$ we denote the adjugate matrix, i.e., the matrix such that $A A^{*}=$ $A^{*} A=\operatorname{det}(A) I_{n}$ (note that for a generic matrix $M=\left(x_{i j}\right)$ the entries of the matrix $M^{*}$ are homogeneous polynomials in $\left\{x_{i j}\right\}$ of degree $\left.n-1\right)$;

for a map $f: X \rightarrow Y$ and a subset $S \subset X$ we denote by $\operatorname{Res}_{S} f$ the restriction of $f$ to $S$. 
Let $X$ be an algebraic variety defined over $K$, and let $\left\{X_{i}\right\}$ be a countable set of proper closed subsets $X_{i} \varsubsetneqq X$. Then we call the set $X \backslash \cup_{i} X_{i}$ a quasi-open subset of $X$. (In topology and real analysis such sets are often called " $G_{\delta}$-sets".)

If $X$ is an algebraic variety and $Y \subset X$, then $\bar{Y}$ is the Zariski closure of $Y$ in $X$.

\section{WORD MAPS WITH CONSTANTS}

Let $\widetilde{w}_{\Sigma}: G^{m} \rightarrow G$ be a word map with constants of a simple algebraic group $G$. Note that there are words with constants $w_{\Sigma}$ such that $\operatorname{Im} \widetilde{w}_{\Sigma}=1$ (so-called identities with constants, see, e.g., G2]). Such identities exist if and only if the root system of $G$ contains roots of different length. However, even in the cases when all roots are of the same length we cannot expect the analogue of the Borel Theorem $\overline{\operatorname{Im} \widetilde{w}_{\Sigma}}=G$. It would be interesting to understand the influence of the properties of the set $\Sigma$ on the dimension of $\overline{\operatorname{Im} w_{\Sigma}}$. In such a generality, this seems to be a difficult question, and we start with considering some particular situations.

Theorem 1.1. Let $w_{1}, \ldots, w_{r+1} \in F_{m}$ be words where $w_{2}, \ldots, w_{r} \neq 1$. There exists an open set $\mathcal{U} \subset G^{r}$ such that for every $\Sigma=\left(\sigma_{1}, \ldots, \sigma_{r}\right) \in \mathcal{U}$ and for the word with constants $w_{\Sigma}=w_{1} \sigma_{1} w_{2} \sigma_{2} \cdots w_{r} \sigma_{r} w_{r+1}$, the dimension $\operatorname{dim} \overline{\operatorname{Im} \widetilde{w}_{\Sigma}}$ is a fixed number $\mathfrak{d}=$ $\mathfrak{d}\left(w_{1}, \ldots, w_{r+1}\right)$ depending only on $w_{1}, \ldots, w_{r+1} \in F_{m}$. Moreover,

$$
\operatorname{dim} \overline{\operatorname{Im} \widetilde{w}_{\Sigma^{\prime}}} \leq \mathfrak{d}
$$

for every $\Sigma^{\prime}=\left(\sigma_{1}^{\prime}, \ldots, \sigma_{r}^{\prime}\right) \in G^{r}$ (here we admit the possibility $\left.\sigma_{i}^{\prime} \in Z(G)\right)$.

Proof. Define the word

$$
w^{Y}=w_{1} y_{1} w_{2} y_{2} \cdots w_{r} y_{r} w_{r+1} \in F_{m+r}=\left\langle x_{1}, \ldots, x_{m}, y_{1}, \ldots, y_{r}\right\rangle .
$$

We have dominant maps

$$
\widetilde{w}^{Y}: G^{m+r} \rightarrow G \text { and } p_{Y}: G^{m+r} \rightarrow G^{r}
$$

where $p_{Y}$ is the projection onto the components $m+1, \ldots, m+r$. Consider the map

$$
\Phi: G^{m+r} \stackrel{\left(\widetilde{w}^{Y}, p_{Y}\right)}{\rightarrow} G \times G^{r} .
$$

Let $X=\overline{\operatorname{Im} \Phi} \subset G \times G^{r}$, and let $p_{Y}^{\prime}: X \rightarrow G^{r}$ be the projection onto $G^{r}$. Then $p_{Y}^{\prime}(X)=G^{r}$ because for every $r$-tuple $\Sigma=\left(\sigma_{1}, \ldots, \sigma_{r}\right) \in G^{r}$ there is a non-empty set

$$
Z_{\Sigma}=\left\{\left(\widetilde{w}_{\Sigma}\left(g_{1}, \ldots, g_{m}\right), \sigma_{1}, \ldots, \sigma_{r}\right) \mid\left(g_{1}, \ldots, g_{m}\right) \in G^{m}\right\} \subset X .
$$

One can show that there exists an open subset $\mathcal{V}$ of $X$ such that:

(a) $\mathcal{V} \subset \operatorname{Im} \Phi$,

(b) for every $v \in \mathcal{V}$ the dimension of every irreducible component of the preimage $\Phi^{-1}(v)$ is a fixed number $\mathfrak{f}$,

(c) for every $u \in \operatorname{Im} \Phi$ the dimension of every irreducible component of the preimage $\Phi^{-1}(u)$ is greater than or equal to $\mathfrak{f}$ 
(cf., e.g., [Shaf, Chapter I, n. 6, Th. 7]). Let now $\mathcal{U} \subset G^{r}$ be an open subset contained in $p_{Y}^{\prime}(\mathcal{V})$, and let $\Sigma=\left(\sigma_{1}, \ldots, \sigma_{r}\right) \in \mathcal{U}$. Let $v \in \mathcal{V}$ be such that $p_{Y}^{\prime}(v)=\Sigma$. Then $v=\left(\widetilde{w}_{\Sigma}\left(g_{1}, \ldots, g_{m}\right), \sigma_{1}, \ldots, \sigma_{r}\right)$ for some $\left(g_{1}, \ldots, g_{m}\right) \in G^{m}$, and the dimension of every irreducible component of the preimage $\Phi^{-1}(v)$ is equal to $\mathfrak{f}$, see (b). Further, the Zariski closure $\bar{Z}_{\Sigma}$ is an irreducible closed subset of $X$. Indeed, $Z_{\Sigma}$ is the image of an irreducible variety under the morphism $\Phi_{\Sigma}: G^{m} \rightarrow G \times G^{r}$ given by the formula $\Phi_{\Sigma}\left(x_{1}, \ldots, x_{m}\right)=\left(w_{\Sigma}\left(x_{1}, \ldots, x_{m}\right), \sigma_{1}, \ldots, \sigma_{r}\right)$ (in other words, $\Phi_{\Sigma}$ is the restriction of $\Phi$ to $\left.G^{m} \times\left(\sigma_{1}, \ldots, \sigma_{r}\right) \subset G^{m} \times G^{r}\right)$. Note that $v \in Z_{\Sigma} \cap \mathcal{V}$. Hence there is an open subset $\mathcal{W}$ of $\bar{Z}_{\Sigma}$ such that $v \in \mathcal{W} \subset \mathcal{V}$. Since $\mathcal{W} \subset \mathcal{V}$, the dimension of every irreducible component of $\Phi^{-1}\left(v^{\prime}\right)$ for every point $v^{\prime} \in \mathcal{W}$ is equal to $\mathfrak{f}$, see (b). Also, for every $v^{\prime} \in \mathcal{W}$ the closed subset $\Phi^{-1}\left(v^{\prime}\right) \subset G^{m} \times G^{r}$ is isomorphic (as an affine variety) to the closed subset $\Phi_{\Sigma}^{-1}\left(v^{\prime}\right) \subset G^{m}$. Hence the dimension of the general fibre of the morphism $\Phi_{\Sigma}: G^{m} \rightarrow G \times G^{r}$ is equal to $\mathfrak{f}$, and therefore

$$
\operatorname{dim} \overline{\operatorname{Im} \Phi_{\Sigma}}=m \operatorname{dim} G-\mathfrak{f} .
$$

The construction of $\Phi_{\Sigma}$ shows that $\overline{\operatorname{Im} \Phi_{\Sigma}}$ is isomorphic to $\overline{\operatorname{Im} \widetilde{w}_{\Sigma}}$ (the projection of $G \times G^{r}$ onto the first component gives this isomorphism). Hence $\operatorname{dim} \overline{\operatorname{Im} \widetilde{w}_{\Sigma}}=m \operatorname{dim} G-\mathfrak{f}$ for every $\Sigma \in \mathcal{U}$.

Let $\Sigma^{\prime}=\left(\sigma_{1}^{\prime}, \ldots, \sigma_{r}^{\prime}\right) \in G^{r}$ (possibly, $\sigma_{i}^{\prime} \in Z(G)$ for some $i$ ). Note that over the points of $G \times \Sigma \subset G \times G^{r}$ the maps $\widetilde{w}_{\Sigma^{\prime}}: G^{m} \rightarrow G$ and $\Phi_{\Sigma^{\prime}}: G^{m} \rightarrow G \times G^{r}$ have the same fibres. Moreover, these fibres are also fibres of the map $\Phi: G^{m} \times G^{r} \rightarrow G \times G^{r}$ which correspond to points of the form $\left(\widetilde{w}_{\Sigma^{\prime}}\left(g_{1}, \ldots, g_{m}\right), \sigma_{1}^{\prime}, \ldots, \sigma_{r}^{\prime}\right)$. Since the dimension of every fibre of $\Phi$ is at least $\mathfrak{f}$ (see $(\mathrm{c})$ ), the dimension $\mathfrak{f}^{\prime}$ of the general fibre of $\widetilde{w}_{\Sigma^{\prime}}$ is at least $\mathfrak{f}$. Hence

$$
\operatorname{dim} \overline{\operatorname{Im} \widetilde{w}_{\Sigma^{\prime}}}=m \operatorname{dim} G-\mathfrak{f}^{\prime} \leq \mathfrak{d}=m \operatorname{dim} G-\mathfrak{f} .
$$

Definition 1.2. Given an $(r+1)$-tuple of words $\Omega_{r}=\left(w_{1}, \ldots, w_{r+1}\right)$ of $F_{m}$ where $w_{2}, \ldots, w_{r} \neq 1$, we say that $\Sigma=\left(\sigma_{1}, \ldots, \sigma_{r}\right)$ is regular for $\Omega_{r}$ if $\Sigma \in \mathcal{U}$ where $\mathcal{U}$ is an open subset satisfying the conditions of Theorem [1.1.

Example 1.3. Let $m=1$ and $\Omega_{2}=\left\{w_{1}=x, w_{2}=x^{-1}\right\}$. Then for every $\Sigma=\{\sigma\}$ the image of $\widetilde{w}_{\Sigma}$ is the conjugacy class of $\sigma$, and therefore for a regular $\Sigma$ the dimension of $\operatorname{Im} \widetilde{w}_{\Sigma}$ is equal to the dimension of the conjugacy class of a regular element $\sigma$ of $G$, that is, $\operatorname{dim} G-\operatorname{rank} G$.

Corollary 1.4. Let $\Omega_{r}=\left(w_{1}, \ldots, w_{r+1}\right)$ be such that $w_{2}, \ldots, w_{r} \neq 1$. Suppose $\prod_{i=1}^{r+1} w_{i} \neq$ 1. Then if $\Sigma=\left(\sigma_{1}, \ldots, \sigma_{r}\right)$ is regular for $\Omega_{r}$, the map $\widetilde{w}_{\Sigma}: G^{m} \rightarrow G$ is dominant.

Proof. Indeed, for $\Sigma=(1,1, \ldots, 1)$ we have $w_{\Sigma}=w=\prod_{i=1}^{r+1} w_{i} \neq 1$, and therefore $\widetilde{w}_{\Sigma}$ is dominant according to Borel's Theorem. Now the statement immediately follows from Theorem 1.1.

Remark 1.5. Since the point $(1,1, \ldots, 1)$ is the most peculiar case, presumably, the condition $w=\prod_{i=1}^{r+1} w_{i} \neq 1$ is sufficient for every $\widetilde{w}_{\Sigma}$ to be dominant. 
Below we consider one important example where the word with constants is obtained by substitution of an element of $G$ instead of one variable and where the condition $\prod_{i=1}^{r+1} w_{i} \neq$ 1 of Corollary 1.4 may not hold (such words with constants are used in [G3]).

Let $w\left(x_{1}, \ldots, x_{m}, y\right) \in F_{m+1}$,

$$
w=w_{1} y^{k_{1}} w_{2} y^{k_{2}} w_{3} \cdots w_{r} y^{k_{r}} w_{r+1}
$$

where $w_{1}, \ldots, w_{r+1} \in F_{m}$ and $w_{2}, \ldots, w_{r} \neq 1$. Then for every $\sigma \in G$ we have the word with constants

$$
w_{\sigma}=w_{1} \sigma^{k_{1}} w_{2} \sigma^{k_{2}} w_{3} \cdots w_{r} \sigma^{k_{r}} w_{r+1}
$$

(actually, according to the definition, we have to exclude the cases where $\sigma^{k_{i}} \in Z(G)$ for some $i$ ).

Theorem 1.6. Suppose $\sum_{j=1}^{m} k_{j}=0$. Then there exists an open subset $\mathcal{V} \subset G$ such that for any $\sigma \in \mathcal{V}$ the map

$$
\pi \circ \widetilde{w}_{\sigma}: G^{m} \rightarrow T / W
$$

is dominant.

Proof. Choose a sequence $G_{1}<G_{2}<\cdots<G_{\ell}=G$ of simple algebraic subgroups of $G$ and a sequence $T_{1} \leq \cdots \leq T_{\ell}=T$ of their maximal tori so that $\operatorname{rank} G_{i}=i$ and all the $G_{i}$ are $T$-invariant.

This can be done as follows. Let $\left\{\alpha_{1}, \ldots, \alpha_{\ell}\right\}$ be an irreducible root system corresponding to $G$. We may assume that the simple roots $\alpha_{i}$ are numbered so that for every $i \leq \ell$ the set $\left\{\alpha_{1}, \ldots, \alpha_{i}\right\}$ is an irreducible root system. Let $G_{i}$ be the corresponding subgroup of $G$ (generated by the root subgroups of the root system $\left\{\alpha_{1}, \ldots, \alpha_{i}\right\}$ ). Then we have $G_{1}<G_{2}<\cdots<G_{\ell}=G$. Further, let $T_{i}=\left\{h_{\alpha_{1}}\left(t_{1}\right) h_{\alpha_{2}}\left(t_{2}\right) \cdots h_{\alpha_{i}}\left(t_{i}\right) \mid t_{1}, \ldots, t_{i} \in K^{*}\right\}$ be the corresponding maximal torus of $G_{i}$ (here $\left\{h_{\alpha_{i}}(t) \mid t \in K^{*}\right\}$ is the corresponding one-dimensional torus in the simple algebraic group generated by the root subgroups $\left.X_{ \pm \alpha_{i}}\right)$ ), and let $T=T_{\ell}$. Then we have $T_{1}<T_{2}<\cdots<T_{\ell}=T$. Obviously, $T$ normalizes every root subgroup $X_{\beta}, \beta \in\left\langle\alpha_{1}, \ldots, \alpha_{\ell}\right\rangle$, and therefore it normalizes every $G_{i}$. Then in the reductive group $G_{i} T$ we have $T=T_{i} H_{i}$ where $H_{i}$ is the subtorus of $T$ which centralizes $G_{i}$. Let $\pi_{i}: G_{i} \rightarrow T_{i} / W_{i}$ denote the quotient morphisms.

The embedding $\iota: T_{i-1} \rightarrow T_{i}$ induces a morphism of varieties

$$
\iota^{*}: T_{i-1} / W_{i-1} \rightarrow T_{i} / W_{i} .
$$

Obviously, the morphism $\iota^{*}$ is quasi-finite (every non-empty fibre is finite). Then

$$
\operatorname{dim} \overline{\iota^{*}\left(T_{i-1} / W_{i-1}\right)}=\operatorname{dim} T_{i-1}=i-1 .
$$

The set $\overline{\iota^{*}\left(T_{i-1} / W_{i-1}\right)}$ is an irreducible codimension one subset of $T_{i} / W_{i}$.

Now suppose that $\sigma \in T$. Recall that for every $i$ we have $T=T_{i} H_{i}$ where $H_{i}$ is the centralizer of $G_{i}$ in $T$. Hence $\sigma=\sigma_{i} h_{i}$ for some $\sigma_{i} \in T_{i}, h_{i} \in H_{i}$. Then for every $m$-tuple $\left(g_{1}, \ldots, g_{m}\right) \in G_{i}^{m}$ we have

$$
w_{\sigma}\left(g_{1}, \ldots, g_{m}\right)=w\left(g_{1}, \ldots, g_{m}, \sigma_{i}\right) h_{i}^{\sum_{j} k_{j}}=w\left(g_{1}, \ldots, g_{m}, \sigma_{i}\right) \in G_{i}
$$

(recall that $\sum_{j=1}^{r} k_{j}=0$ ). Hence the restriction of $\widetilde{w}_{\sigma}$ to $G_{i}^{m}$ gives the map

$$
\widetilde{w}_{\sigma}^{i}: G_{i}^{m} \rightarrow G_{i}
$$


Lemma 1.7. For every $1<i \leq \ell$ there is an element $\sigma \in T$ such that

$$
\overline{\pi_{i}\left(\operatorname{Im} \widetilde{w}_{\sigma}^{i}\right)} \nsubseteq \overline{\iota^{*}\left(T_{i-1} / W_{i-1}\right)} \text {. }
$$

Proof. Assume to the contrary that $\overline{\pi_{i}\left(\operatorname{Im} \widetilde{w}_{\sigma}^{i}\right)} \subseteq \overline{\iota^{*}\left(T_{i-1} / W_{i-1}\right)}$ for every $\sigma \in T$. Then for every $m$-tuple $\left(g_{1}, \ldots, g_{m}\right) \in G_{i}^{m}$ and every $\sigma_{i} \in T_{i}$ we have

$$
\pi_{i} \circ \widetilde{w}\left(g_{1}, \ldots, g_{m}, \sigma_{i}\right) \subset \overline{\iota^{*}\left(T_{i-1} / W_{i-1}\right)} .
$$

Note that any $(m+1)$-tuple of semisimple elements $\left(\gamma_{1}, \ldots, \gamma_{m}, \gamma\right)$ is conjugate by an element of $G_{i}$ to an $(m+1)$-tuple of the form $\left(g_{1}, \ldots, g_{m}, \sigma_{i}\right)$ where $\sigma_{i} \in T$. Since the set of $(m+1)$-tuples $\left(\gamma_{1}, \ldots, \gamma_{m}, \gamma\right)$ of semisimple elements is dense in $G_{i}^{m+1}$ and the map $\pi_{i} \circ \widetilde{w}$ is invariant under conjugation by elements of $G_{i}$, we have $\pi_{i} \circ \widetilde{w}\left(G_{i}^{m+1}\right) \subset \overline{\iota^{*}\left(T_{i-1} / W_{i-1}\right)}$, and therefore

$$
\operatorname{dim} \overline{\pi_{i} \circ \widetilde{w}\left(G_{i}^{m+1}\right)} \leq i-1 .
$$

This implies that $\overline{\pi_{i} \circ \widetilde{w}\left(G_{i}^{m+1}\right)} \neq \overline{\pi_{i}\left(G_{i}\right)}$, which is a contradiction with the Borel Theorem [Bo1].

Lemma 1.8. For every $1<i \leq \ell$ there is a non-empty open subset $S_{i} \subset T$ such that

$$
\overline{\pi_{i}\left(\operatorname{Im} \widetilde{w}_{\sigma}^{i}\right)} \nsubseteq \overline{\iota^{*}\left(T_{i-1} / W_{i-1}\right)}
$$

for every $\sigma \in S_{i}$.

Proof. Let $\omega^{i}: G_{i}^{m} \times T \rightarrow G_{i}$ be given by the formula

$$
\omega_{i}\left(g_{1}, \ldots, g_{m}, \sigma\right)=\widetilde{w}_{\sigma}^{i}\left(g_{1}, \ldots, g_{m}\right)
$$

Consider the composite map

$$
\pi_{i} \circ \omega^{i}: G_{i}^{m} \times T \stackrel{\omega^{i}}{\rightarrow} G_{i} \stackrel{\pi_{i}}{\rightarrow} T_{i} / W_{i}
$$

Let $X=\left(\pi_{i} \circ \omega^{i}\right)^{-1}\left(\overline{\left.\iota^{*}\left(T_{i-1} / W_{i-1}\right)\right)}\right.$. Then $X$ is a proper closed subset in $G_{i}^{m} \times T$ by Lemma 1.7. Let $X=\cup_{q} X_{q}$ be the decomposition of $X$ into the union of irreducible closed subsets. Further, let $i_{T}: X \rightarrow T$ be the map induced by the projection $G_{i}^{m} \times T \rightarrow T$. Suppose $\overline{i_{T}\left(X_{q}\right)} \neq T$ for some $q$. Put $S_{i q}=T \backslash \overline{i_{T}\left(X_{q}\right)}$. Now, if $\sigma \in S_{i q}$ then $i_{T}^{-1}(\sigma) \cap X_{q}=\emptyset$. Suppose $\overline{i_{T}\left(X_{q}\right)}=T$ for some $q$. Since $\operatorname{dim} X_{q}<\operatorname{dim} G_{i}^{m} \times T$, there is a non-empty open subset $S_{i q} \subset T$ such that $\operatorname{dim} i_{T}^{-1}(\sigma) \cap X_{q}<\operatorname{dim} G_{i}^{m}$ for every $\sigma \in S_{i q}$. Thus, if $\sigma \in S_{i}=\cap_{q} S_{i q}$, the set $G_{i}^{m} \times\{\sigma\}$ is not contained in $X$ and therefore $\overline{\pi_{i}\left(\operatorname{Im} \widetilde{w}_{\sigma}^{i}\right)} \nsubseteq$ $\iota^{*}\left(T_{i-1} / W_{i-1}\right)$.

Lemma 1.9. There is a non-empty open subset $S \subset T$ such that for every $1<i \leq \ell$ and every $\sigma \in S$ we have

$$
\left.\overline{\pi_{i}\left(\operatorname{Im} \widetilde{w}_{\sigma}^{i}\right)} \nsubseteq \overline{\iota^{*}\left(T_{i-1} / W_{i-1}\right.}\right)
$$

Proof. Take $S=\cap_{i} S_{i}$ where $S_{i}$ is an open set from Lemma 1.8,

We can now prove the theorem. Choose $S$ as in Lemma 1.9. Let $\sigma \in S$. Suppose

$$
\overline{\pi_{i-1}\left(\operatorname{Im} \widetilde{w}_{\sigma}^{i-1}\right)}=T_{i-1} / W_{i-1} .
$$


Note that for $i=1$ we have $T_{0}=\{1\}, W_{0}=\{1\}$ and therefore for $i=1$ assumption (1.2) holds. Since for every $i$ the map $\widetilde{w}_{\sigma}^{i}$ is the restriction of $\widetilde{w}_{\sigma}$ to $G_{i}^{m}$ (see (1.1)), we have

$$
\pi_{i}\left(\operatorname{Im} \widetilde{w}_{\sigma}^{i-1}\right) \subset \pi_{i}\left(\operatorname{Im} \widetilde{w}_{\sigma}^{i}\right) .
$$

Since $\pi_{i}\left(\operatorname{Im} \widetilde{w}_{\sigma}^{i-1}\right) \subset T_{i} / W_{i}$ is the image of $\pi_{i-1}\left(\operatorname{Im} \widetilde{w}_{\sigma}^{i-1}\right) \subset T_{i-1} / W_{i-1}$ with respect to the map $\iota^{*}: T_{i-1} / W_{i-1} \rightarrow T_{i} / W_{i}$, we obtain from (1.2) and the assumption on $\sigma$ that

$$
\overline{\iota^{*}\left(T_{i-1} / W_{i-1}\right)} \varsubsetneqq \overline{\pi_{i}\left(\operatorname{Im} \widetilde{w}_{\sigma}^{i}\right)} .
$$

Since $\overline{\pi_{i}\left(\operatorname{Im} \widetilde{w}_{\sigma}^{i}\right)}$ and $\overline{\iota^{*}\left(T_{i-1} / W_{i-1}\right)}$ are closed irreducible subsets of $T_{i} / W_{i}$ and $\operatorname{dim} \overline{\iota^{*}\left(T_{i-1} / W_{i-1}\right)}$ $=i-1$, we have

$$
\overline{\pi_{i}\left(\operatorname{Im} \widetilde{w_{\sigma}^{i}}\right)}=T_{i} / W_{i} .
$$

The induction step now finishes the proof.

Remark 1.10. Presumably, the assumption $\sum_{j=1}^{r} k_{j}=0$ can be replaced with a weaker condition: the word $w\left(x_{1}, \ldots, x_{m}, y\right) \in F_{m+1}$ is not of the form $\omega y^{l} \omega^{-1}$ for some $\omega=$ $\omega\left(x_{1}, \ldots, x_{m}, y\right) \in F_{m+1}$. However, the example $w(x, \sigma)=x \sigma x^{-1}$ shows that we cannot avoid some restrictions on the word $w$.

Remark 1.11. It would be interesting to extend our considerations to words with constants and automorphisms (anti-automorphisms) of $G$ where we have variables $x_{i}$ and variables $x_{i}^{\varphi_{i}}$ marked by automorphisms. Such a word also gives rise to a map $G^{m} \rightarrow G$. Note that given a word with constants $w_{\Sigma}$ and a collection $\Phi=\left\{\varphi_{i}\right\}(i=1, \ldots, m)$ of automorphisms of $G$, there are (at least) two natural ways to produce such a map: first replace each appearance of $x_{i}^{a_{i}}$ in $w_{\Sigma}$ with $\left(x_{i}^{\varphi_{i}}\right)^{a_{i}}$, then either

(1) replace each such expression with $\varphi_{i}\left(g_{i}\right)^{a_{i}}$, or

(2) do this only in the cases where the exponent $a_{i}$ is positive,

and compute the resulting value in $G$. Although the second option might seem a little artificial, it would include (at least) two important cases: 1) twisted conjugacy classes, see, e.g., [Sp], [FT] and the references therein for a survey of various aspects of this theory, and 2) twisted commutators, see, e.g., [Se], [NS1], [NS2], [Le].

\section{WORD MAPS AND CENTRAL FUNCTIONS}

General definitions. Let $G=\mathrm{SL}_{n}(K)$. Then we extend a word map with constants $\widetilde{w}_{\Sigma}: G^{m} \rightarrow G$ to a map

$$
\widetilde{w}_{\Sigma}^{*}: \mathrm{M}_{n}^{m}(K) \rightarrow \mathrm{M}_{n}(K)
$$

in the following way. Let

$$
w_{\Sigma}=w_{1} \sigma_{1} w_{2} \sigma_{2} \cdots w_{r} \sigma_{r} w_{r+1}=x_{i_{1}}^{a_{1}} x_{i_{2}}^{a_{2}} \cdots x_{i_{s_{1}}}^{a_{s_{1}}} \sigma_{1} x_{i_{s_{1}+1}}^{a_{s_{1}+1}} \cdots x_{i_{s_{2}}}^{a_{s_{2}}} \sigma_{2} x_{i_{s_{2}+1}}^{a_{s_{2}+1}} \cdots
$$

be a word map with constants where $\Sigma=\left\{\sigma_{1}, \ldots, \sigma_{r}\right\} \subset \mathrm{SL}_{n}(K)$. For any $m$-tuple of matrices $\left(\mu_{1}, \ldots, \mu_{m}\right) \in \mathrm{M}_{n}^{m}(K)$ we define

$$
\widetilde{w}_{\Sigma}^{*}\left(\mu_{1}, \ldots, \mu_{m}\right) \in \mathrm{M}_{n}(K)
$$


by putting in formula (2.1) instead of each expression $x_{i_{k}}^{a_{k}}$ the matrix $\bar{\mu}_{i_{k}}^{\left|a_{k}\right|}$ where

$$
\bar{\mu}_{i_{k}}=\left\{\begin{array}{ll}
\mu_{i_{k}} & \text { if } a_{k}>0 \\
\mu_{i_{k}}^{*} & \text { if } a_{k}<0
\end{array} .\right.
$$

In other words, we substitute in formula (2.1) matrices instead of variables replacing each negative exponent with its module and in each such case replacing the given matrix with the adjugate matrix.

Remark 2.1. The word map with constants $\widetilde{w}_{\Sigma}: \mathrm{SL}_{n}^{m}(K) \rightarrow \mathrm{SL}_{n}(K)$ admits a natural extension to the group $\mathrm{GL}_{n}(K)$. Below we also denote by $\widetilde{w}_{\Sigma}$ the corresponding map $\mathrm{GL}_{n}^{m}(K) \rightarrow \mathrm{GL}_{n}(K)$.

Note that the map $\widetilde{w}_{\Sigma}^{*}$ can be represented by an $n \times n$-matrix whose entries are polynomial functions in the entries of the matrices $\mu_{i}$. More precisely:

Proposition 2.2. $\quad$ (i) Let $A=K\left[\left\{y_{p q}^{r}\right\}_{r=1}^{m}\right]$ be the ring of polynomial functions on $\mathrm{M}_{n}^{m}$ where $y_{p q}^{r}$ corresponds to the $(p, q)^{\text {th }}$ entry of the $r^{\text {th }}$ component of $\mathrm{M}_{n}^{m}$ (here $1 \leq p, q \leq n, 1 \leq r \leq m)$. Further, let

$$
a=\sum_{a_{k}>0} a_{k}, \quad b=\sum_{a_{k}<0}\left|a_{k}\right|
$$

Then

$$
\widetilde{w}_{\Sigma}^{*}=\left(\begin{array}{cccc}
w_{11} & w_{12} & \cdots & w_{1 n} \\
w_{21} & w_{22} & \cdots & w_{2 n} \\
\cdots & & & \\
w_{n 1} & w_{2 n} & \cdots & w_{n n}
\end{array}\right) \in \mathrm{M}_{n}(A)
$$

where each $w_{i j}$ is a homogeneous polynomial in $\left\{y_{p q}^{r}\right\}$ of degree $a+(n-1) b$.

(ii) Let $\Delta_{r}$ be the homogeneous polynomial of degree $n$ in the variables $y_{p q}^{r}$ given by the determinant of the $r^{\text {th }}$ component of $\mathrm{M}_{n}$, and let

$$
\Delta=\prod_{r=1}^{m} \Delta_{r}^{b_{r}} \text { where } b_{r}=\sum_{a_{k}<0, i_{k}=r}\left|a_{k}\right| .
$$

Then, restricting to $\mathrm{GL}_{n}$ and $\mathrm{SL}_{n}$, we obtain, respectively

$$
\operatorname{Res}_{\mathrm{GL}_{n}} \widetilde{w}_{\Sigma}^{*}=\Delta \widetilde{w}_{\Sigma} \text { and } \operatorname{Res}_{\mathrm{SL}_{n}} \widetilde{w}_{\Sigma}^{*}=\widetilde{w}_{\Sigma}
$$

Proof. Straightforward from the definition.

Example 2.3. Let $m=1, n=2$,

$$
\Sigma=\left\{\sigma_{1}=\sigma=\left(\begin{array}{cc}
s & 0 \\
0 & s^{-1}
\end{array}\right), \sigma_{2}=\sigma^{-1}=\left(\begin{array}{cc}
s^{-1} & 0 \\
0 & s
\end{array}\right) \quad \mid s \neq 1\right\},
$$

$w_{\Sigma}=\sigma x \sigma^{-1} x^{-1}$. Then $a=1, b=1$, and $\widetilde{w}_{\Sigma}^{*}$ is a function on $\mathrm{M}_{2}$ which can be expressed by the formula

$$
\widetilde{w}_{\Sigma}^{*}=\left(\begin{array}{cc}
s & 0 \\
0 & s^{-1}
\end{array}\right)\left(\begin{array}{ll}
y_{11} & y_{12} \\
y_{21} & y_{22}
\end{array}\right)\left(\begin{array}{cc}
s^{-1} & 0 \\
0 & s
\end{array}\right)\left(\begin{array}{cc}
y_{22} & -y_{12} \\
-y_{21} & y_{11}
\end{array}\right)=
$$




$$
=\left(\begin{array}{cc}
y_{11} y_{22}-s^{2} y_{12} y_{21} & y_{11} y_{12}\left(s^{2}-1\right) \\
y_{21} y_{22}\left(1-s^{-2}\right) & y_{11} y_{22}-s^{-2} y_{12} y_{21}
\end{array}\right) \text {. }
$$

Central functions on $\operatorname{Im}_{\widetilde{w}_{\Sigma}^{*}}$. Let $X \in \mathrm{M}_{n}(K)$, and let

$$
\chi(X)=\lambda^{n}+\chi_{1}(X) \lambda^{n-1}+\cdots+\chi_{n}(X)
$$

be the characteristic polynomial of $X$. Then the map $\chi_{i}: \mathrm{M}_{n}(K) \rightarrow K, X \mapsto \chi_{i}(X)$, is given by a homogeneous polynomial of degree $i$ in the entries of $X$.

The following fact should be compared with [BZ, Lemma 2.1].

Theorem 2.4. Let

$$
\widetilde{w}_{\Sigma}: \mathrm{SL}_{n}^{m}(K) \rightarrow \mathrm{SL}_{n}(K) .
$$

Then $\chi_{i} \circ \widetilde{w}_{\Sigma}: \mathrm{SL}_{n}^{m}(K) \rightarrow K$ is either a constant function, or takes every value.

Proof. We start with the following lemma.

Lemma 2.5. Let $F$ be a polynomial function on $\mathrm{M}_{n}^{m}(K)$ which is homogeneous on each component of $\mathrm{M}_{n}^{m}(K)$, that is, there exist natural $d_{i}, i=1, \ldots, m$, such that

$$
F\left(g_{1}, \ldots, g_{i-1}, c g_{i}, g_{i+1}, \ldots, g_{m}\right)=c^{d_{i}} F\left(g_{1}, \ldots, g_{i-1}, g_{i}, g_{i+1}, \ldots, g_{m}\right)
$$

for every $\left(g_{1}, \ldots, g_{m}\right) \in \mathrm{M}_{n}^{m}(K)$ and every $c \in K$. Then the restriction of $F$ to $\mathrm{SL}_{n}^{m}(K)$ is either a constant function, or takes every value.

Proof. Suppose that the restriction of $F$ to $\mathrm{SL}_{n}^{m}(K)$ is not a constant function. Let $\alpha \in K$, and denote by $X_{\alpha}$ the hypersurface in the affine space $\mathrm{M}_{n}^{m}(K)$ defined by the equation

$$
F^{n}-\alpha \prod_{r=1}^{m} \Delta_{r}^{d_{r}}=0 .
$$

(Here $A=K\left[\left\{y_{p q}^{r}\right\}_{r=1}^{m}\right], \Delta_{r}, \Delta$ are defined as in Proposition 2.2.) Denote by $X_{0}$ the hypersurface of $\mathrm{M}_{n}^{m}(K)$ given by the equation $\prod_{r=1}^{m} \Delta_{r}=0$. If $X_{\alpha} \subset X_{0}$, then

$$
F^{n}-\alpha \prod_{r=1}^{m} \Delta_{r}^{d_{r}}=\beta \Delta_{i_{1}}^{e_{i_{1}}} \Delta_{i_{2}}^{e_{i_{2}}} \cdots \Delta_{i_{s}}^{e_{i_{s}}}
$$

for some $1 \leq i_{1} \leq i_{2} \leq \cdots \leq i_{s} \leq m, \beta \in K^{*}$ and $e_{i_{j}} \in \mathbb{N}$ (note that each $\Delta_{r}$ is an irreducible polynomial in a polynomial ring $A$ ), and therefore $F^{n}\left(g_{1}, \ldots, g_{m}\right)=\alpha+\beta$ for every $\left(g_{1}, \ldots, g_{m}\right) \in \mathrm{SL}_{n}^{m}(K)$, which contradicts our assumption. Hence, there is $\left(g_{1}, \ldots, g_{m}\right) \in \mathrm{GL}_{n}^{m}(K)$ such that

$$
F^{n}\left(g_{1}, \ldots, g_{m}\right)=\alpha \prod_{r=1}^{m} \Delta_{r}^{d_{r}}\left(g_{r}\right) .
$$

On the other hand, $g_{r}=\sqrt[n]{\Delta_{r}\left(g_{r}\right)} g_{r}^{\prime}$ for some $g_{r}^{\prime} \in \mathrm{SL}_{n}(K)$. Now, using the condition on $F$ we obtain

$$
F^{n}\left(g_{1}, \ldots, g_{m}\right)=F^{n}\left(g_{1}^{\prime}, \ldots, g_{m}^{\prime}\right) \prod_{r=1}^{m} \Delta_{r}^{d_{r}}\left(g_{r}\right) .
$$

Hence $F^{n}\left(g_{1}^{\prime}, \ldots, g_{m}^{\prime}\right)=\alpha$. Thus, the function $F^{n}$ takes every value on $\operatorname{SL}_{n}^{m}(K)$. The same is of course true for the function $F$. 
Let now define $F=\chi_{i} \circ \widetilde{w}_{\Sigma}^{*}: \mathrm{M}_{n}^{m}(K) \rightarrow K$. Then $F$ satisfies the assumptions of Lemma 2.5 (see Proposition 2.2). Hence $\operatorname{Res}_{\mathrm{SL}_{n}^{m}} F$ is either a constant function, or takes every value. We have

$$
\operatorname{Res}_{\mathrm{SL}_{n}^{m}} F=\operatorname{Res}_{n}^{m} \chi_{i} \circ \widetilde{w}_{\Sigma}^{*}=\chi_{i} \circ \operatorname{ResSL}_{n}^{m} \widetilde{w}_{\Sigma}^{*}=\chi_{i} \circ \widetilde{w}_{\Sigma},
$$

which proves the theorem.

Corollary 2.6. If

$$
\chi_{1} \circ \widetilde{w}_{\Sigma}: \mathrm{SL}_{2}^{m}(K) \rightarrow \mathrm{SL}_{2}(K)
$$

is not a constant function, then every non-central semisimple conjugacy class of $\mathrm{SL}_{2}(K)$ intersects $\operatorname{Im} \widetilde{w}_{\Sigma}$.

Proof. Let $C$ be a non-central semisimple conjugacy class of $\mathrm{SL}_{2}(K)$, and let $\alpha=\chi_{1}(g)=$ $\operatorname{tr} g$ for $g \in C$. Further, let $\left(g_{1}, \ldots, g_{m}\right) \in \mathrm{SL}_{2}^{m}(K)$ be such that $\operatorname{tr}\left(\widetilde{w}_{\Sigma}\left(g_{1}, \ldots, g_{m}\right)\right)=\alpha$. Then $\widetilde{w}_{\Sigma}\left(g_{1}, \ldots, g_{m}\right) \in C$.

This result gives rise to an alternative proof of the following theorem by Bandman and Zarhin.

Corollary 2.7 (cf. [BZ]). The image of a non-trivial word map $\widetilde{w}: \mathrm{SL}_{2}^{m}(K) \rightarrow \mathrm{SL}_{2}(K)$ contains every semisimple element except, possibly, -1 .

Proof. We may view the word $w$ as $w_{\Sigma}$ with $\Sigma=\emptyset$. Also, the identity element 1 is always in $\operatorname{Im} w$.

Here is another corollary.

Theorem 2.8. Let $G$ be a simple algebraic group. Suppose that $G$ is not of type $A_{r}, r>1$, $D_{2 k+1}, k>1$, or $E_{6}$, and let $\widetilde{w}: G^{m} \rightarrow G$ be a non-trivial word map. Then every regular semisimple element of $G$ is contained in $\operatorname{Im} \widetilde{w}$. Moreover, for every semisimple $g \in G$ there exists $g_{0} \in G$ of order $\leq 2$ such that $g g_{0} \in \operatorname{Im} \widetilde{w}$.

Proof. Let $R$ be an irreducible root system of rank $r$. Let us check the property: there exists a root subsystem $R^{\prime} \subset R$ such that

$$
R^{\prime}=R_{1} \cup R_{2} \cup \cdots \cup R_{r} \subset R \text { where } R_{i}=A_{1} .
$$

Property $\left(^{*}\right)$ implies that

$$
G_{1} \times G_{2} \times \cdots \times G_{r} \leq G_{R} \text { where } G_{i}=S L_{2}(K), P G L_{2}(K)
$$

The following fact is apparently well known (one can extract it, e.g., from [GOV, Table 5 on page 234]). We present a proof for the sake of completeness. We use the notation of Bou] throughout.

Lemma 2.9. Property (*) holds for all irreducible root systems except for $A_{r}, r>1$, $D_{2 k+1}, k>1$, and $E_{6}$.

Proof. Case $A_{r}$. Obviously, (**) does not hold $\left(\mathrm{SL}_{2}^{r} \nless \mathrm{SL}_{r+1}\right)$. Then $\left(^{*}\right)$ does not hold for $r>1$.

Case $B_{r}$. We have $R=\left\langle\mathfrak{e}_{1}-\mathfrak{e}_{2}, \ldots, \mathfrak{e}_{r-1}-\mathfrak{e}_{r}, e_{r}\right\rangle$. 
Subcase $r=2 m+1$. We have $R_{1}=\left\langle\mathfrak{e}_{1}-\mathfrak{e}_{2}\right\rangle, R_{2}=\left\langle\mathfrak{e}_{1}+\mathfrak{e}_{2}\right\rangle, R_{3}=\left\langle\mathfrak{e}_{3}-\mathfrak{e}_{4}\right\rangle, R_{4}=$ $\left\langle\mathfrak{e}_{3}+\mathfrak{e}_{4}\right\rangle, \ldots, R_{2 m}=\left\langle\mathfrak{e}_{2 m-1}+\mathfrak{e}_{2 m}\right\rangle, R_{2 m+1}=\left\langle\mathfrak{e}_{2 m+1}\right\rangle$.

Subcase $r=2 m$. We have $R_{1}=\left\langle\mathfrak{e}_{1}-\mathfrak{e}_{2}\right\rangle, R_{2}=\left\langle\mathfrak{e}_{1}+\mathfrak{e}_{2}\right\rangle, R_{3}=\left\langle\mathfrak{e}_{3}-\mathfrak{e}_{4}\right\rangle, R_{4}=\left\langle\mathfrak{e}_{3}+\right.$ $\left.\mathfrak{e}_{4}\right\rangle, \ldots, R_{2 m}=\left\langle\mathfrak{e}_{2 m-1}+\mathfrak{e}_{2 m}\right\rangle$.

Case $C_{r}$. We have $R_{i}=\left\langle 2 \mathfrak{e}_{i}\right\rangle$ (long roots).

Case $D_{2 m}$. The same as $B_{2 m}$.

Case $D_{2 m+1}$. Consider the standard representation of $D_{2 m+1}$ with weights $\pm \mathfrak{e}_{1}, \ldots, \pm \mathfrak{e}_{2 m+1}$. For a root $\alpha=\mathfrak{e}_{i} \pm \mathfrak{e}_{j}$, the semisimple root subgroup $h_{\alpha}(t)$ acts non-trivially exactly on the four weight vectors corresponding to $\pm \mathfrak{e}_{i}, \pm \mathfrak{e}_{j}$. Then if $\left(^{*}\right)$ holds, we can divide the dimension of the representation by 4 . But this dimension equals $2(2 m+1)$, so (*) does not hold for $r=2 m+1$.

Case $\mathbf{E}_{6}$. In $E_{6}$ there are no six mutually orthogonal positive roots. Indeed, since all roots lie in the same $W$-orbit, we may start with

$$
\alpha=\frac{1}{2}\left(\mathfrak{e}_{1}+\mathfrak{e}_{2}+\mathfrak{e}_{3}+\mathfrak{e}_{4}+\mathfrak{e}_{5}-\mathfrak{e}_{6}-\mathfrak{e}_{7}+\mathfrak{e}_{8}\right) .
$$

There are altogether fifteen positive roots orthogonal to $\alpha$ : ten roots of the form

$$
\beta=-\mathfrak{e}_{i}+\mathfrak{e}_{j}, 1 \leq i<j \leq 5,
$$

and five roots of the form

$$
\gamma=\frac{1}{2}\left( \pm \mathfrak{e}_{1} \pm \mathfrak{e}_{2} \pm \mathfrak{e}_{3} \pm \mathfrak{e}_{4} \pm \mathfrak{e}_{5}-\mathfrak{e}_{6}-\mathfrak{e}_{7}+\mathfrak{e}_{8}\right)
$$

(where the number of minus signs is equal to 4 ). The set of roots of the form $\beta$ contains orthogonal subsets of size at most two, and the set of roots of the form $\gamma$ contains no orthogonal subsets. Hence one can find at most three mutually orthogonal positive roots, and thus $(*)$ does not hold.

Case $E_{7}$. Here $D_{6} \cup\left\langle\mathfrak{e}_{7}-\mathfrak{e}_{8}\right\rangle \subset R$, and therefore $\left(^{*}\right)$ holds.

Case $E_{8}$. Here $D_{8} \subset E_{8}$, and therefore $(*)$ holds.

Case $F_{4}$. Here $D_{4} \subset F_{4}$, and therefore $(*)$ holds.

Case $G_{2}$. Here $\left\langle\mathfrak{e}_{1}-\mathfrak{e}_{2}\right\rangle \cup\left\langle-2 \mathfrak{e}_{3}+\mathfrak{e}_{1}+\mathfrak{e}_{2}\right\rangle \subset G_{2}$, and therefore $(*)$ holds.

We can now prove the theorem. Let $\Gamma_{i}=\mathrm{SL}_{2}(K)$, and let

$$
\Psi: \prod_{i=1}^{r} \Gamma_{i} \rightarrow G
$$

be the natural homomorphism induced by the inclusion $(* *)$.

Denote by $\widetilde{w}_{i}$ the word map $\Gamma_{i}^{m} \rightarrow \Gamma_{i}$ defined by the same word $w$. Then $\operatorname{Im} \widetilde{w}_{i}$ contains every semisimple element of $\Gamma_{i}$ except, possibly, -1 .

Let $T_{i}$ be a maximal torus of $\Gamma_{i}$. 
We have $\Psi\left(\prod_{i=1}^{r} T_{i}\right)=T$. (Indeed, $\Psi\left(\prod_{i=1}^{r} T_{i}\right) \subset T$, and $\Psi\left(\prod_{i=1}^{r} T_{i}\right)$ is a torus of dimension $r=\operatorname{dim} T$.) Let $t \in T$. Then

$$
t=\Psi\left(t_{1}, t_{2}, \ldots, t_{r}\right) \text { for some } t_{i} \in T_{i} .
$$

First suppose that $t_{i} \neq-1$ for every $i$. Then

$$
t_{i}=\widetilde{w}_{i}\left(g_{i 1}, \ldots, g_{i m}\right) \text { for some }\left(g_{i 1}, \ldots, g_{i m}\right) \in \Gamma_{i}^{m},
$$

and therefore

$$
t=\widetilde{w}\left(\gamma_{1}, \ldots, \gamma_{m}\right) \text { where } \gamma_{j}=\Psi\left(g_{1 j}, g_{2 j}, \ldots, g_{r j}\right)
$$

Note that the condition $t_{i} \neq-1$ holds for all regular semisimple elements $t$ (indeed, if $t_{i}=-1$, then $\Psi\left(1, \ldots, 1, u_{i}, 1 \ldots 1\right) \neq 1$ commutes with $t$ for a non-trivial unipotent element $u_{i} \in \Gamma_{i} \approx \mathrm{SL}_{2}(K)$ ). Then all such elements lie in $\operatorname{Im} \widetilde{w}$.

If $t_{i}=-1$ for some $i$, then we may take

$$
t_{0}=\left(t_{1}^{\prime}, t_{2}^{\prime}, \ldots, t_{r}^{\prime}\right) \text { where } t_{i}^{\prime}=\left\{\begin{array}{l}
-1, \text { if } t_{i}=-1 \\
1 \text { if } t_{i} \neq-1
\end{array} .\right.
$$

Then the order of $\Psi\left(t_{0}\right)$ is at most two, and $\Psi\left(t t_{0}\right) \in \operatorname{Im} \widetilde{w}$.

Remark 2.10. Presumably, Theorem 2.4 can be extended in the following way. Instead of one function $\chi \circ \widetilde{w}$ one could consider the map $\pi \circ \widetilde{w}$ where $\pi=\left(\chi_{1}, \ldots, \chi_{n-1}\right): \mathrm{SL}_{n} \rightarrow$ $T / W=\mathbb{A}^{n-1}$ is the quotient map. Say, for a tuple $\alpha=\left(\alpha_{1}, \ldots, \alpha_{n-1}\right) \in T / W$ one can consider the system of equations

$$
\left[\chi_{i} \circ \widetilde{w}^{*}(Y)\right]^{n}-\alpha_{i} \prod_{r=1}^{m} \Delta_{r}^{d_{i r}}(Y)=0
$$

where $d_{i r}$ is the homogeneous degree of $\chi_{i} \circ \widetilde{w}^{*}(Y)$ with respect to the variables $y_{i j}^{r}$ (see Proposition 2.2, Lemma 2.5). Obviously, this system has solutions in $\mathrm{M}_{n}^{m}(K)$. If the variety of these solutions is not contained in the variety $\prod_{r=1}^{m} \Delta_{r}=0$, we can find a solution in $\mathrm{GL}_{n}^{m}(K)$ of the system $\chi_{i} \circ \widetilde{w}(Y)-\sqrt[n]{\alpha_{i}}=0$ for some $Y \in \mathrm{SL}_{n}^{m}(K)$.

Remark 2.11. Note that if we could prove that $\pi \circ \widetilde{w}$ is a surjective map, we would have every regular semisimple element of any simple group $G$ in $\operatorname{Im} \widetilde{w}$. Indeed, in every irreducible root system of rank $r$ there is a subsystem of rank $r$ which is a union of systems $A_{i}$ (see, e.g., [Bo1]).

\section{REPRESENTATION VARIETIES AND GENERIC GROUPS}

General constructions (see, e.g., [LM], [Pl], [PBK], [Si]).

Let $\Gamma=\left\langle g_{1}, \ldots, g_{m}\right\rangle$ be a finitely generated group, and let $\mathfrak{R}_{\Gamma} \subset F_{m}$ be the set of all relations of $\Gamma$.

Put

$$
R(\Gamma, G)=\left\{\left(x_{1}, \ldots, x_{m}\right) \in G^{m} \quad \mid \omega\left(x_{1}, \ldots, x_{m}\right)=1 \text { for every } \omega \in \mathfrak{R}_{\Gamma}\right\} .
$$

Obviously, $R(\Gamma, G)$ is a Zariski closed subset of $G^{m}$ (which is defined over $K$ ), and for every $\left(x_{1}, \ldots, x_{m}\right) \in R(\Gamma, G)$ the subgroup $\left\langle x_{1}, \ldots, x_{m}\right\rangle \leq G$ is a quotient of $\Gamma$. One can identify the sets

$$
R(\Gamma, G)=\operatorname{Hom}(\Gamma, G)
$$


using the one-to-one correspondence

$$
\left(x_{1}, \ldots, x_{m}\right) \in R(\Gamma, G) \leftrightarrow \rho \in \operatorname{Hom}(\Gamma, G)
$$

given by

$$
\left(\rho\left(g_{1}\right), \ldots, \rho\left(g_{m}\right)\right)=\left(x_{1}, \ldots, x_{m}\right) .
$$

The set $R\left(\Gamma, \mathrm{GL}_{n}(K)\right)$ is called the variety of $n$-dimensional representations of $\Gamma$.

The "variety" $R(\Gamma, G)$ may be non-reduced and reducible, so the scheme language is most appropriate, see, e.g., Si]. However, we will freely use the abusive term "variety" in what follows.

Let $R(\Gamma, G)=\cup_{i} R(\Gamma, G)^{i}$ be the decomposition into the union of irreducible closed subsets. Then we have the following property for the components.

Proposition 3.1. Let $K$ be an algebraically closed field of infinite transcendence degree over a prime subfield. Then for each $i$ there exists a dense quasi-open subset $U^{i} \subset R(\Gamma, G)^{i}$ such that for every $\left(g_{1}, \ldots, g_{m}\right) \in U^{i}$ the subgroup $\left\langle g_{1}, \ldots, g_{m}\right\rangle$ is isomorphic to a fixed quotient of $\Gamma$.

Proof. For any $\omega \in F_{m}$ the set

$$
X_{\omega}=\left\{\left(g_{1}, \ldots, g_{m}\right) \in G^{m} \quad \mid \omega\left(g_{1}, \ldots, g_{m}\right)=1\right\}
$$

is a proper closed subset of $G^{m}$. Hence $R(\Gamma, G)^{i} \cap X_{\omega}$ is a closed subset of $R(\Gamma, G)^{i}$. Let now

$$
Q_{i}=\left\{\omega \in F_{m} \quad \mid \quad R(\Gamma, G)^{i} \nsubseteq X_{\omega}\right\} .
$$

Then

$$
\bigcup_{\omega \in Q_{i}} R(\Gamma, G)^{i} \cap X_{\omega}
$$

is a countable union of proper closed subsets of $R(\Gamma, G)^{i}$. Since $K$ is of infinite transcendence degree over a prime subfield, we have

$$
U^{i}=R(\Gamma, G)^{i} \backslash \bigcup_{\omega \in Q_{i}}\left(R(\Gamma, G)^{i} \cap X_{\omega}\right) \neq \emptyset
$$

(see [Bo2]), and therefore $U^{i}$ is a dense quasi-open subset of $R(\Gamma, G)^{i}$. Every group $\left\langle g_{1}, \ldots, g_{m}\right\rangle$ for $\left(g_{1}, \ldots, g_{m}\right) \in U^{i}$ has the same set of relations: $\left\{\omega \in F_{n} \mid R(\Gamma, G)^{i} \subset\right.$ $\left.X_{\omega}\right\}$.

Definition 3.2. A group isomorphic to $\left\langle g_{1}, \ldots, g_{m}\right\rangle$ for $\left(g_{1}, \ldots, g_{m}\right) \in U^{i}$ will be called the generic group of the component $R(\Gamma, G)^{i}$.

For every $\rho \in R(\Gamma, G)$ and every $g \in G$ the map $\rho_{g}: \Gamma \rightarrow G$ given by $\rho_{g}(\gamma)=g \rho(\gamma) g^{-1}$ is also an element of $R(\Gamma, G)$. Hence we have a regular action of the algebraic group $G$ on the affine set $R(\Gamma, G)$. If $G=\mathrm{GL}_{n}(K)$ or $\mathrm{SL}_{n}(K)$, orbits correspond to classes of equivalent representations. In these cases (or, more generally, if $G$ is a reductive group) there exists a categorical quotient $R(\Gamma, G) / / G$ which is also a closed affine set. There is a one-to-one correspondence between points of $R\left(\Gamma, \mathrm{GL}_{n}(K)\right) / / \mathrm{GL}_{n}(K)\left(\operatorname{resp} . R\left(\Gamma, \mathrm{SL}_{n}(K)\right) / / \mathrm{SL}_{n}(K)\right)$ and classes of completely reducible $n$-dimensional representations of $\Gamma$ (see [LM]). 
Let $\mathfrak{B}_{\Gamma} \subset \mathfrak{R}_{\Gamma}$ be a minimal set of relations (that is, $\mathfrak{B}_{\Gamma}$ is a minimal set of generators of the group $\mathfrak{R}_{\Gamma}$ as a normal subgroup of $\left.F_{m}\right)$. Since the equality $\omega\left(x_{1}, \ldots, x_{m}\right)=1$, where $x_{1}, \ldots, x_{m} \in G, \omega \in F_{m}$, implies that $\omega\left(y x_{1} y^{-1}, \ldots, y x_{m} y^{-1}\right)=1$ for every $y \in G$, we may reduce the set of all relations $\mathfrak{R}_{\Gamma}$ in (3.1) to the set $\mathfrak{B}_{\Gamma}$. Let us now assume that $\mathfrak{B}_{\Gamma}=\left\{\omega_{1}, \ldots, \omega_{k}\right\}$ is a finite set. Then we may consider the map

$$
\Phi_{\mathfrak{B}_{\Gamma}}: G^{m} \rightarrow G^{k}
$$

defined by

$$
\Phi_{\mathfrak{B}_{\Gamma}}\left(x_{1}, \ldots, x_{m}\right)=\left(\omega_{1}\left(x_{1}, \ldots, x_{m}\right), \ldots, \omega_{k}\left(x_{1}, \ldots, x_{m}\right)\right) .
$$

Then

$$
R(\Gamma, G)=\Phi_{\mathfrak{B}_{\Gamma}}^{-1}\left(e_{k}\right)
$$

(here $e_{k}=(1, \ldots, 1)$ is the identity of $\left.G^{k}\right)$, see [LM].

Finitely generated one-relator groups. Let $\mathfrak{B}_{\Gamma}=\{w\}$. Then we will write $\Gamma_{w}$ instead of $\Gamma$ to emphasize the relation $w$.

Further, here $k=1$ and the map $\Phi_{\mathfrak{B}_{\Gamma}}: G^{m} \rightarrow G$ is the word map

$$
\widetilde{w}: G^{m} \rightarrow G .
$$

In this case we denote

$$
\widetilde{w}^{-1}(1)=\mathcal{W}_{w}
$$

From (3.2) we have $\mathcal{W}_{w}=R\left(\Gamma_{w}, G\right)$. Denote by $\mathcal{W}_{w}^{i}$ the irreducible components of $\mathcal{W}_{w}$ where $i=0,1, \ldots$.

In what follows, the image of the identity element 1 of a fixed torus $T$ of $G$ in the quotient variety $T / W$ is also denoted by 1 . Define

$$
\mathcal{T}_{w}=(\pi \circ \widetilde{w})^{-1}(1)
$$

and denote by $\mathcal{T}_{w}^{j}$ the irreducible components of $\mathcal{T}_{w}$ where $j=0,1, \ldots$ Then the following simple statement is of key importance.

\section{Proposition 3.3.}

(a) For every irreducible component $\mathcal{W}_{w}^{i}$ of $\mathcal{W}_{w}$ there is an irreducible component $\mathcal{T}_{w}^{j}$ of $\mathcal{T}_{w}$ which contains $\mathcal{W}_{w}^{i}$.

(b) If $\mathcal{W}_{w} \neq \mathcal{T}_{w}$, then $\operatorname{Im} \widetilde{w}$ contains a non-trivial unipotent element $u$.

Proof.

(a) Obviously, $\mathcal{W}_{w} \subset \mathcal{T}_{w}$ and hence every irreducible component of $\mathcal{W}_{w}$ is contained in an irreducible component of $\mathcal{T}_{w}$.

(b) Indeed, the set $\mathcal{T}_{w} \subset G^{m}$ is exactly the set of $m$-tuples $\left(g_{1}, \ldots, g_{m}\right) \in G^{m}$ such that $w\left(g_{1}, \ldots, g_{m}\right)$ is a unipotent element.

Remark 3.4. Presumably, in (b) one can replace "if" with "if and only if". 
If $K$ is a field of infinite transcendence degree over a prime subfield, then for each $i$ there exists a dense quasi-open subset $U^{i} \subset \mathcal{W}_{w}^{i}(K)$ such that for every $\left(g_{1}, \ldots, g_{m}\right) \in U^{i}$ the subgroup $\left\langle g_{1}, \ldots, g_{m}\right\rangle$ is isomorphic to a fixed quotient of $\Gamma_{w}$ (Proposition 3.1).

The generic group $\left\langle g_{1}, \ldots, g_{m}\right\rangle$, where $\left(g_{1}, \ldots, g_{m}\right) \in U^{i}$, will be denoted by $\widetilde{\Gamma}_{w}^{i}$. The question on describing the possibilities for $\widetilde{\Gamma}_{w}^{i}$ for a given group $\Gamma_{w}$ is interesting in its own right. More specifically, answering it could help in describing words $w$ with the condition $\mathcal{W}_{w} \neq \mathcal{T}_{w}$, which guarantees the existence of non-trivial unipotent elements in Im $\widetilde{w}$. Below we consider some examples when $\mathcal{W}_{w} \neq \mathcal{T}_{w}$.

\section{EXAMPLES OF $R\left(\Gamma_{w}, \mathrm{SL}_{2}(\mathbb{C})\right)$}

In this section we use the following notation:

$G=\mathrm{SL}_{2}(\mathbb{C})$;

$T, B, B^{-}, U, U^{-}$are the sets of diagonal, upper and lower triangular, and upper and lower unitriangular matrices;

$\dot{w}_{0}$ is an element of $N_{G}(T) \backslash T$;

$w \in F_{2}$;

$R\left(\Gamma_{w}, \mathrm{SL}_{2}(\mathbb{C})\right)=\mathcal{W}_{w}=\bigcup_{i=1}^{l} \mathcal{W}_{w}^{i}$ is the decomposition into the union of irreducible components;

$\mathcal{T}_{w}$ is the hypersurface in $G \times G$ defined by the equation $\operatorname{tr} w(x, y)=2$;

$\mathcal{T}_{w}=\bigcup_{j=1}^{e} \mathcal{T}_{w}^{j}$ is the decomposition into the union of irreducible components.

Obviously, for every $j$ we have

$$
\operatorname{dim} \mathcal{T}_{w}^{j}=5
$$

Also, for every $i=1, \ldots, l$ there is $j=1, \ldots, e$ such that $\mathcal{W}_{w}^{i} \subset T_{w}^{j}$ and

$$
3 \leq \operatorname{dim} \mathcal{W}_{w}^{i} \leq 5
$$

(Indeed, the upper inequality follows from (4.1). Since $\mathcal{W}_{w}$ is defined by three equations $w_{11}=1, w_{12}=w_{21}=0$, where

$$
\left.\widetilde{w}(x, y)=\left(\begin{array}{ll}
w_{11}(x, y) & w_{12}(x, y) \\
w_{21}(x, y) & w_{22}(x, y)
\end{array}\right) \quad \mid x, y \in G\right\}
$$

the dimension of every component of $\mathcal{W}_{w}$ is at least $\operatorname{dim}(G \times G)-3=6-3=3$.)

Note that according to Proposition 3.3.

$$
\operatorname{dim} \mathcal{W}_{w}^{i} \leq 4 \Rightarrow \text { there exists a non-trivial unipotent element } u \in \operatorname{Im} \widetilde{w} .
$$

We start with some simple examples.

Example 4.1. $\mathrm{w}=[\mathrm{x}, \mathrm{y}]$.

In this case $\mathcal{W}_{w}$ is classically known as the commuting variety of $G$. See, e.g., [RBKC] and the references therein for its properties and related problems.

Proposition 4.2. The set $\mathcal{W}_{w}$ is irreducible, $\operatorname{dim} \mathcal{W}_{w}=4$ and

$$
\left.\mathcal{W}_{w}=\overline{\left\{g(T \times T) g^{-1}\right.} \mid g \in G\right\} .
$$

The set $\mathcal{T}_{w}$ is also irreducible, $\operatorname{dim} \mathcal{T}_{w}=5$, and

$$
\left.\mathcal{T}_{w}=\overline{\left\{g(B \times B) g^{-1}\right.} \mid g \in G\right\} .
$$


Proof. The irreducibility of the commuting variety in $G \times G$ has been proven in [Ri] in a more general case where $G$ is a semisimple simply connected group. A general pair in $\mathcal{W}_{w}$ is a pair of commuting semisimple elements. Hence we have (4.3). Since the general $G$-orbit (under conjugation) of a pair $\left(t_{1}, t_{2}\right)$ is of $\operatorname{dimension} \operatorname{dim} G / T=2$, we have $\operatorname{dim} \mathcal{W}_{w}=4$.

Let $\left(g_{1}, g_{2}\right) \in \mathcal{T}_{w}^{j}$ be a general pair. We may assume $\pm 1 \neq g_{2}=t \in T$ (since $\operatorname{dim} \mathcal{T}_{w}^{j}=5$, it cannot happen that all pairs $\left(g_{1}, g_{2}\right)$ consist of \pm unipotent elements). Show that $g_{1} \in B \cup B^{-}$. Assume the contrary. Then either $g_{1}=v s u$ for some $1 \neq v \in U^{-}, 1 \neq u \in$ $U, s \in T$, or $g_{1}=\dot{w}_{0} u$ for some $\dot{w}_{0}$ and $u \in U$. In the first case,

$$
[v s u, t]=v s u t u^{-1} s^{-1} v^{-1} t^{-1}=v u^{\prime} v^{\prime} \text { for some } v^{-1} \neq v^{\prime} \in U^{-}, 1 \neq u^{\prime} \in U \text {. }
$$

Indeed, since both $s$ and $t$ are diagonal matrices in $\mathrm{SL}_{2}$ and $t \neq \pm 1$, we have $t x t^{-1} \neq x$ for every $x \in U$ or $x \in U^{-}$, hence

$$
\text { vsutu }{ }^{-1} s^{-1} v^{-1} t^{-1}=v s \underbrace{\underbrace{u\left(t u^{-1} t^{-1}\right)}_{\in U, \neq 1}}_{:=u^{\prime} \in U, \neq 1} s^{-1} \underbrace{\left(t v^{-1} t^{-1}\right)}_{:=v^{\prime} \in U^{-}, \neq v^{-1}}=v u^{\prime} v^{\prime} .
$$

Hence $[v s u, t] \sim v^{\prime \prime} u^{\prime}$ for some $1 \neq v^{\prime \prime} \in U^{-}, 1 \neq u^{\prime} \in U$. But an element of the form $v^{\prime \prime} u^{\prime}$ cannot have the trace equal to 2 . This is a contradiction.

In the second case, we have $g_{1}=\dot{w}_{0} u$. Then $\operatorname{tr}\left[\dot{w}_{0} u, t\right]=\operatorname{tr} t^{-2} \neq 2$. This is also a contradiction.

Thus, we have a pair $\left(g_{1}, g_{2}\right)$ where $\pm 1 \neq g_{1} \in T$ and $g_{2} \in B \cup B^{-}$. Hence $\left(g_{1}, g_{2}\right) \in$ $\left\{g(B \times B) g^{-1} g \in G\right\}$. This implies that

$$
\left.\mathcal{T}_{w}^{j} \subset \overline{\left\{g(B \times B) g^{-1} \mid\right.} g \in G\right\} .
$$

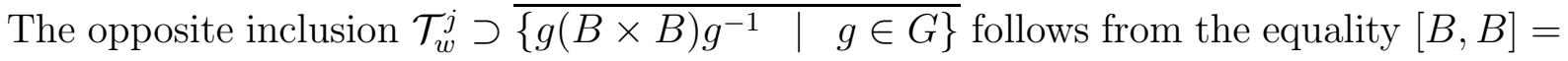
$U$.

Example 4.3. $\mathrm{w}=\left[\mathrm{x}^{\mathrm{m}}, \mathrm{y}^{\mathrm{n}}\right]$.

Denote

$$
\mathfrak{m}=\{k \in \mathbb{N}|2<k| 2 m\}, \quad \mathfrak{n}=\{l \in \mathbb{N}|2<l| 2 n\} .
$$

Let $C_{r} \subset G$ be the conjugacy class of elements of order $r$.

Proposition 4.4. We have

$$
\mathcal{W}_{w}^{0}=\mathcal{W}_{[x, y]} \subset \mathcal{T}_{w}^{0}=\mathcal{T}_{[x, y]} .
$$

All other irreducible components are of one of the following forms:

$$
\mathcal{W}_{w}^{j}=\mathcal{T}_{w}^{j}=C_{j} \times G
$$

for $j \in \mathfrak{m}$ or

$$
\mathcal{W}_{w}^{j}=\mathcal{T}_{w}^{j}=G \times C_{j}
$$

for $j \in \mathfrak{n}$. 
Proof. The existence of the components $\mathcal{W}_{w}^{0}=\mathcal{W}_{[x, y]} \subset \mathcal{T}_{w}^{0}$ is obvious.

Let now $(x, y) \in \mathcal{W}_{w}^{j}$ be such that $x^{m} \neq \pm 1, y^{n} \neq \pm 1$. Then the elements $x, x^{m}$ are either in the same torus of $G$ or in the same unipotent subgroup (modulo the centre). The same is true for $y, y^{n}$. Since $\left[x^{m}, y^{n}\right]=1$ we then have $[x, y]=1$ and therefore $(x, y) \in \mathcal{W}_{w}^{0}$. Thus, if $(x, y) \in \mathcal{W}_{w}^{j} \backslash \mathcal{W}_{w}^{0}$ then either $x^{m}= \pm 1$ or $y^{n}= \pm 1$ and $\mathcal{W}_{w}^{j}$ is one of the components for $j \in \mathfrak{m}$ or $j \in \mathfrak{n}$ which have been pointed out in the statement. Since the components $\mathcal{W}_{w}^{j}, j>1$, are isomorphic to the direct product of a conjugacy class $C_{g}$ for a semisimple element $g$ of order $>2$ and the group $G$, we have $\operatorname{dim} \mathcal{W}_{w}^{j}=5$ and therefore $\mathcal{W}_{w}^{j}=\mathcal{T}_{w}^{j}$.

Definition 4.5. We say that a subgroup $H \leq G$ is a free product modulo the centre if $H / Z(G) \approx R * Q$ for some $R, Q \leq G / Z(G)$. In this case we write

$$
H=R * Q \bmod Z(G)
$$

Now for the integer $j$ put

$$
[j]=\left\{\begin{array}{l}
j \text { if } j \text { is odd } \\
\frac{j}{2} \text { if } j \text { is even }
\end{array}\right.
$$

Proposition 4.6. For $j>0$ we have

$$
\widetilde{\Gamma}_{w}^{j}=\mathbb{Z}_{[j]} * \mathbb{Z} \bmod Z(G) \text { or } \widetilde{\Gamma}_{w}^{j}=\mathbb{Z} * \mathbb{Z}_{[j]} \bmod Z(G)
$$

where $\mathbb{Z}$ is the infinite cyclic group and $\mathbb{Z}_{[j]}$ is the cyclic group of order $[j]$.

Proof. For any two non-central conjugacy classes $C_{1}, C_{2} \subset G$, the generic group $\left\langle g_{1}, g_{2}\right|$ $\left.g_{1} \in C_{1}, g_{2} \in C_{2}\right\rangle$ is isomorphic to $\left\langle g_{1}\right\rangle *\left\langle g_{2}\right\rangle \bmod Z(G)$, see [G2]. Hence the statement follows from Proposition 4.4.

Example 4.7. $\mathrm{w}=[\mathrm{x}, \mathrm{y}]^{2}$.

Let

$$
t=\left(\begin{array}{cc}
\lambda & 0 \\
0 & \lambda^{-1}
\end{array}\right), g=\left(\begin{array}{cc}
\alpha & \beta \\
\gamma & \delta
\end{array}\right) \in G
$$

We have

$$
[t, g]=\left(\begin{array}{cc}
\alpha \delta-\beta \gamma \lambda^{2} & \alpha \beta\left(-1+\lambda^{2}\right) \\
\gamma \delta\left(-1+\lambda^{-2}\right) & \alpha \delta-\beta \gamma \lambda^{-2}
\end{array}\right)
$$

Hence

$$
\operatorname{tr}([t, g])=2-\beta \gamma\left(\lambda-\lambda^{-1}\right)^{2} .
$$

Thus,

$$
\operatorname{tr}([t, g])=a \Leftrightarrow 2-\beta \gamma\left(\lambda-\lambda^{-1}\right)^{2}=a \Leftrightarrow \beta \gamma=\frac{2-a}{\left(\lambda-\lambda^{-1}\right)^{2}}:=p_{a, \lambda} .
$$

Since $\operatorname{det} g=1$, we have

$$
\beta \gamma=\frac{2-a}{\left(\lambda-\lambda^{-1}\right)^{2}} \Leftrightarrow \alpha \delta=1+\frac{2-a}{\left(\lambda+\lambda^{-1}\right)^{2}}=\frac{\lambda^{2}+\lambda^{-2}-a}{\left(\lambda-\lambda^{-1}\right)^{2}}:=q_{a, \lambda} .
$$


Put

$$
T_{a}:=\left\{t=\left(\begin{array}{cc}
\lambda & 0 \\
0 & \lambda^{-1}
\end{array}\right), \lambda \neq \pm 1, \lambda^{2}+\lambda^{-2}-a \neq 0\right\} .
$$

If $t \in T_{a}, a \neq 2$, then

$$
M_{t}^{a}=\left\{\left(\begin{array}{ll}
\alpha & \beta \\
\gamma & \delta
\end{array}\right) \in G \quad \mid \beta \gamma=p_{a, \lambda}\right\}=\left\{\left(\begin{array}{ll}
\alpha & \beta \\
\gamma & \delta
\end{array}\right) \quad \mid \quad \beta \gamma=p_{a, \lambda}, \alpha \delta=q_{a, \lambda}\right\}
$$

is an irreducible closed subset in $G \times G$ and $\operatorname{dim} M_{t}^{a}=2$. The construction of $M_{t}^{a}$ implies that

$$
M_{T}^{a}:=\left\{\left(t, M_{t}^{a}\right) \mid t \in T_{a}\right\}=\left\{(t, g) \mid t \in T_{a}, g \in G, \operatorname{tr}([t, g])=a\right\} .
$$

Also, the set $M_{T}^{a}$ is an irreducible locally closed subset of $G \times G$, and $\operatorname{dim} M_{T}^{a}=3$. Now let $\Psi: M_{T}^{a} \times G \rightarrow G \times G$ be defined by $\Psi(t, g, y)=\left(y t y^{-1}, y g y^{-1}\right)$. Since $M_{T}^{a}$ is an irreducible locally closed subset of $G \times G$ and $G$ is an affine variety, the closure of the image of $\Psi$ is an irreducible closed subset of $G$. Thus, the set

$$
S_{a}:=\overline{\operatorname{Im} \Psi}=\overline{\left\{g M_{T}^{a} g^{-1} \mid g \in G\right\}}=\overline{\left\{\left(g t g^{-1}, g M_{t}^{a} g^{-1}\right) \mid t \in T_{a}, g \in G\right\}}
$$

is an irreducible closed subset of $G \times G$. Further, the projection $p: S_{a} \rightarrow G$ onto the first component of $G \times G$ is dominant because the image is invariant under conjugation and contains every $t \in T_{a}$. The fibre $p^{-1}(t)$ is equal to $M_{t}^{a}$ and therefore is of dimension 2 . Hence

$$
\operatorname{dim} S_{a}=5
$$

Note that we also have (4.11) and the irreducibility of $S_{2}$ by Proposition 4.2 .

We need the following irreducibility statement, which is probably known to experts. Following the referee's suggestion, we provide a self-contained proof.

Lemma 4.8. Let $a \in \mathbb{C}$. Then the set $\left\{\left(g_{1}, g_{2}\right) \quad \mid \operatorname{tr}\left(\left[g_{1}, g_{2}\right]\right)=a\right\}$ is irreducible and

$$
\left\{\left(g_{1}, g_{2}\right) \mid \operatorname{tr}\left(\left[g_{1}, g_{2}\right]\right)=a\right\}=S_{a} .
$$

Proof. The irreducible closed subset $S_{a}$ is contained in $\left\{\left(g_{1}, g_{2}\right) \quad \mid \operatorname{tr}\left(\left[g_{1}, g_{2}\right]\right)=a\right\}$ (see (4.9), (4.10) ). Equality (4.11) implies that $S_{a}$ is an irreducible component of the set $\left\{\left(g_{1}, g_{2}\right) \mid \operatorname{tr}\left(\left[g_{1}, g_{2}\right]\right)=a\right\}$. Suppose that there exists an irreducible component $S_{a}^{1} \neq S_{a}$ of the set $\left\{\left(g_{1}, g_{2}\right) \mid \operatorname{tr}\left(\left[g_{1}, g_{2}\right]\right)=a\right\}$. Since the set $\left\{\left(g_{1}, g_{2}\right) \quad \mid \operatorname{tr}\left(\left[g_{1}, g_{2}\right]\right)=a\right\}$ is a hypersurface in $\mathrm{SL}_{2}(\mathbb{C}) \times \mathrm{SL}_{2}(\mathbb{C})$, all its irreducible components are of dimension 5 . Thus,

$$
\operatorname{dim} S_{a}^{1}=5 .
$$

Let $p_{1}: S_{a}^{1} \rightarrow G$ be the projection onto the first component of $G \times G$. Since the set $S_{a}^{1}$ is invariant under conjugation by elements of $G$ and it is an irreducible closed subset of $G \times G$, the map $p_{1}$ is either dominant or its image is contained in a single conjugacy class $C$. In the latter case we have $S_{a}^{1}=C \times G$ and $C \neq \pm 1$ (this follows from (4.12)). However, one can find pairs $\left(g_{1}, g_{2}\right),\left(g_{3}, g_{4}\right) \in C \times G$ such that $\left[g_{1}, g_{2}\right]=1,\left[g_{3}, g_{4}\right] \neq 1$ and therefore $\operatorname{tr}\left(\left[g_{1}, g_{2}\right]\right)=2, \operatorname{tr}\left(\left[g_{3}, g_{4}\right]\right) \neq 2$. Thus, the set $S_{a}^{1}$ cannot be of the form $C \times G$. Hence the map $p_{1}$ is dominant and there exists an open subset $T_{a}^{1} \subset T_{a}$ such that $T_{a}^{1} \subset \operatorname{Im} p_{1}$. Now for every $t \in T_{a}^{1}$ we have

$$
S_{a}^{1} \supset\left\{g p_{1}^{-1}(t) g^{-1} \mid t \in T_{a}^{1}, g \in G\right\} \subset\left\{g M_{T}^{a} g^{-1} \mid g \in G\right\} \subset S_{a} .
$$


Since $T_{a}^{1}$ is an open subset of the torus $T$, the set $X=\left\{g p_{1}^{-1}(t) g^{-1} \mid t \in T_{a}^{1}, g \in G\right\}$ contains an open subset of the component $S_{a}^{1}$. But $X$ is also a subset of $S_{a}^{1}$. Thus, $S_{a}=S_{a}^{1}$.

Now we consider our case $w=[x, y]^{2}$.

Since the condition $\operatorname{tr}\left([x, y]^{2}\right)=2$ implies that $[x, y]= \pm u$ where $u$ is a unipotent element, we have $\mathcal{T}_{w}=S_{2} \cup S_{-2}$. The sets $S_{ \pm 2}$ are irreducible of dimension 5 . Thus, $S_{2}=\mathcal{T}_{w}^{0}, S_{-2}=$ $\mathcal{T}_{w}^{1}$. Note that the set $\mathcal{T}_{w}^{0}$ is the variety $\mathcal{T}_{[x, y]}$ considered in Example 4.1. Now consider the set $\mathcal{T}_{w}^{1}$. The definition $T_{a}$ (see (4.7)) implies that

$$
T_{-2}=\left\{t=\left(\begin{array}{cc}
\lambda & 0 \\
0 & \lambda^{-1}
\end{array}\right), \lambda \neq \pm 1, \lambda^{2}+\lambda^{-2}+2 \neq 0\right\}=\left\{t=\left(\begin{array}{cc}
\lambda & 0 \\
0 & \lambda^{-1}
\end{array}\right), \quad \lambda \neq \pm 1, \pm i\right\} .
$$

Then (4.10) implies

$$
\mathcal{T}_{w}^{1}=\overline{\left\{\left(g t g^{-1}, g M_{t}^{-2} g^{-1}\right) \mid t \in T, t^{4} \neq 1, g \in G\right\}}
$$

where $M_{t}^{-2}$ is defined by (4.8). Let $t_{0}=\left(\begin{array}{cc}i & 0 \\ 0 & -i\end{array}\right)$, it is an element of order 4 . Then we can also define $M_{t_{0}}^{-2}$ by formula (4.8) where $p_{a, \lambda}=-1$ and $q_{a, \lambda}=0$ (see (4.5), (4.6) ), namely,

$$
M_{t_{0}}^{-2}=\left\{\left(\begin{array}{ll}
\alpha & \beta \\
\gamma & \delta
\end{array}\right) \quad \mid \beta \gamma=-1, \alpha \delta=0\right\} .
$$

Definitions (4.5), (4.6), (4.8) show that $M_{t_{0}}^{-2}=\{g \in G \mid \operatorname{tr}([t, g])=-2\}$. Hence the set $\left(t_{0}, M_{t_{0}}^{-2}\right)$ is a subset of $S_{-2}$ and therefore we can rewrite the formula for $\mathcal{T}_{w}^{1}$ :

$$
\mathcal{T}_{w}^{1}=\overline{\left\{g t g^{-1} \times g M_{t}^{-2} g^{-1} \mid t \in T, t^{2} \neq 1, g \in G\right\}} .
$$

Proposition 4.9. Each of the sets $\mathcal{T}_{w}$ and $\mathcal{W}_{w}$ has two irreducible components:

$$
\mathcal{W}_{w}^{0}=\mathcal{W}_{[x, y]} \subset \mathcal{T}_{w}^{0}=\mathcal{T}_{[x, y]}, \quad \mathcal{W}_{w}^{1} \subset \mathcal{T}_{w}^{1},
$$

where

$$
\mathcal{W}_{w}^{1}=\left\{g\left(\left(\begin{array}{cc}
i & 0 \\
0 & -i
\end{array}\right),\left(\begin{array}{cc}
0 & \mu \\
-\mu^{-1} & 0
\end{array}\right)\right) g^{-1} \mid \mu \in \mathbb{C}^{*}, g \in G\right\}, \operatorname{dim} \mathcal{W}_{w}^{1}=3
$$

The generic group $\widetilde{\Gamma}_{w}^{1}$ of $\mathcal{W}_{w}^{1}$ is the quaternion group $Q_{8}$.

Proof. We have only one component $\mathcal{W}_{w}^{0}=\mathcal{W}_{[x, y]} \subset \mathcal{T}_{w}^{0}=\mathcal{T}_{w}$ (see Example 4.1).

Consider a pair $\left(g_{1}, g_{2}\right) \in \mathcal{T}_{w}^{1}$ such that $\left[g_{1}, g_{2}\right]=-1$. Then $g_{1} \neq \pm u$ where $u$ is a unipotent element. Indeed, if $g_{1}= \pm u$ we may assume $1 \neq u \in U$ and $g_{2} \in \dot{w}_{0} U$. Let $g_{2}=\dot{w}_{0} u^{\prime}, v \in U$. Then

$$
\left[g_{1}, g_{2}\right]=( \pm u) \dot{w}_{0} u^{\prime}\left( \pm u^{-1}\right) u^{\prime-1} \dot{w}_{0}^{-1}=u \underbrace{\dot{w}_{0}\left(u^{\prime} u^{-1} u^{\prime-1}\right) \dot{w}_{0}^{-1}}_{=v \in U^{-}, v \neq 1}=u v \neq \pm 1
$$

Thus, we may assume

$$
g_{1}=\left(\begin{array}{cc}
\lambda & 0 \\
0 & \lambda^{-1}
\end{array}\right), g_{2}=\left(\begin{array}{cc}
\alpha & \beta \\
\gamma & \delta
\end{array}\right)
$$


where $\lambda \neq \pm 1$. Then formula (4.4) shows that the equality $\left[g_{1}, g_{2}\right]=-1$ is possible if and only if

$$
g_{1}= \pm\left(\begin{array}{cc}
i & 0 \\
0 & -i
\end{array}\right), g_{2}=\left(\begin{array}{cc}
0 & \mu \\
-\mu^{-1} & 0
\end{array}\right)
$$

In these cases $\left\langle g_{1}, g_{2}\right\rangle=Q_{8}$. Since every pair $\left(g_{1}, g_{2}\right)$ with the property $\left[g_{1}, g_{2}\right]=-1$ is conjugate to a pair of the form (4.13), we have only one irreducible component

$$
\mathcal{W}_{w}^{1}=\left\{g\left(\left(\begin{array}{cc}
i & 0 \\
0 & -i
\end{array}\right),\left(\begin{array}{cc}
0 & \mu \\
-\mu^{-1} & 0
\end{array}\right)\right) g^{-1} \mid g \in G\right\} .
$$

If $C_{t_{0}}$ is the conjugacy class of $t_{0}$ then $\mathcal{W}_{w}^{1}$ is the hypersurface in $C_{t_{0}} \times C_{t_{0}}$ given by the equation $[x, y]=-1$. Hence $\operatorname{dim} \mathcal{W}_{w}^{1}=2 \operatorname{dim} C_{t_{0}}-1=3$. Also equality (4.14) shows

$$
\left(g_{1}, g_{2}\right) \in W_{w}^{1} \Leftrightarrow\left\langle g_{1}, g_{2}\right\rangle=Q_{8} .
$$

Example 4.10. $\mathbf{w}=[\mathbf{x}, \mathbf{y}]^{\mathbf{p}}, \mathbf{p} \neq \mathbf{2}$, where $p$ is a prime number.

Proposition 4.11. There are $1+\frac{p-1}{2}$ irreducible components $\mathcal{T}_{w}^{j}$ and the same number of components $\mathcal{W}_{w}^{j} \subset \mathcal{T}_{w}^{j}$. Moreover,

$$
\mathcal{W}_{w}^{0}=\mathcal{W}_{[x, y]} \subset \mathcal{T}_{[x, y]}=\mathcal{T}_{w}^{0}
$$

and for $j=1,2, \ldots, \frac{p-1}{2}$ we have

$$
\mathcal{W}_{w}^{j}=\mathcal{T}_{w}^{j}=\left\{(x, y) \quad \mid \operatorname{tr}([x, y])=2 \cos \frac{2 j \pi}{p}\right\} .
$$

Proof. Obviously, we have

$$
\mathcal{W}_{w}^{0}=\mathcal{W}_{[x, y]} \subset \mathcal{T}_{[x, y]}=\mathcal{T}_{w}^{0}
$$

Let now $\mathcal{T}_{w}^{j} \neq \mathcal{T}_{w}^{0}$. Then

$$
(x, y) \in \mathcal{T}_{w}^{j} \backslash \mathcal{T}_{w}^{0} \Leftrightarrow\left\{\begin{array}{l}
\operatorname{tr}([x, y]) \neq 2 \\
\text { and } \\
{[x, y]^{p}=1 .}
\end{array} \Leftrightarrow[x, y] \backsim\left(\begin{array}{cc}
\epsilon_{p} & 0 \\
0 & \epsilon_{p}^{-1}
\end{array}\right) \quad \text { where } \epsilon_{p}=\sqrt[p]{1} \neq 1 .\right.
$$

The condition $[x, y] \backsim\left(\begin{array}{cc}\epsilon_{p} & 0 \\ 0 & \epsilon_{p}^{-1}\end{array}\right)$ is equivalent to $\operatorname{tr}([x, y])=2 \cos \frac{2 j \pi}{p}$ for some $j=$ $1, \ldots, \frac{p-1}{2}$. Lemma 4.8 shows that there are exactly $\frac{p-1}{2}$ irreducible components $\mathcal{T}_{w}^{j}=$ $\left\{(x, y) \mid \operatorname{tr}([x, y])=2 \cos \frac{2 j \pi}{p}\right\}=S_{2 \cos \frac{2 j \pi}{p}}$ apart from the component $\mathcal{T}_{w}^{0}$. Also, for $j>0$ we have

$$
\mathcal{W}_{w}^{j}=\left\{(x, y) \in \mathcal{T}_{w}^{j} \quad \mid \quad[x, y]^{p}=1\right\}=\mathcal{T}_{w}^{j}
$$

Remark 4.12. The same arguments as in the case $[x, y]^{2}=1$ show that for $j>0$ we have

$$
\mathcal{T}_{w}^{j}=\overline{\left\{g t g^{-1} \times g M_{t}^{2 \cos \frac{2 j \pi}{p}} g^{-1} \mid t \in T, t^{2} \neq 1, g \in G\right\}}
$$


where

$$
M_{t}^{2 \cos \frac{2 j \pi}{p}}=\left\{\left(\begin{array}{ll}
\alpha & \beta \\
\gamma & \delta
\end{array}\right) \in G \quad \mid \beta \gamma=\frac{2\left(1-\cos \frac{2 \pi j}{p}\right)}{\left(\lambda-\lambda^{-1}\right)^{2}}\right\} .
$$

Proposition 4.13. For $j>0$ all $\widetilde{\Gamma}_{w}^{j}$ are non-solvable infinite groups isomorphic to each other.

Proof. A solvable group $\Gamma=\left\langle g_{1}, g_{2}\right\rangle$ in $\mathrm{SL}_{2}(\mathbb{C})$ with the relation $[x, y] \backsim\left(\begin{array}{cc}\epsilon_{p} & 0 \\ 0 & \epsilon_{p}^{-1}\end{array}\right)$ can only be a generalized quaternion group $Q_{4 p}$ (this follows from the classification of subgroups of $\mathrm{SL}_{2}(\mathbb{C})$ ). However, a finite group may only have a finite number of nonequivalent representation in $\mathrm{SL}_{2}(\mathbb{C})$. Hence if $\Gamma$ is the generic group of some irreducible component, then $\Gamma$ is conjugate in $\mathrm{SL}_{2}(\mathbb{C})$ to a fixed subgroup $Q_{4 p} \leq \mathrm{SL}_{2}(\mathbb{C})$. It is easy to see that the dimension of such a component is then equal to 3 , and therefore such a component is a proper closed subset of some $\mathcal{W}_{w}^{j}$. Hence the generic group $\Gamma=\widetilde{\Gamma}_{w}^{j}$ of the component $\mathcal{W}_{w}^{j}$ cannot be solvable.

Let $F \subset \mathbb{C}$ be a pure transcendental extension of $\mathbb{Q}$ of infinite degree, and let $\bar{F} \subset \mathbb{C}$ be its algebraic closure. There exists $\sigma \in \operatorname{Gal}(\bar{F} / F)$ such that $\sigma\left(\epsilon_{p}\right)=\epsilon_{p}^{2}$. Then $\left\{\sigma^{r}\left(\epsilon_{p}\right)\right\}$ where $r=1, \ldots, p-1$, is the set of all roots $\sqrt[p]{1} \neq 1$. Further, the set $\mathcal{W}_{w}$ is $\mathbb{Q}$-defined and therefore $F$-defined. Since $\bar{F}$ is an algebraically closed field of infinite transcendence degree over $\mathbb{Q}$, one can find a pair $\left(g_{1}, g_{2}\right) \in\left(\mathrm{SL}_{2}(\bar{F}) \times \mathrm{SL}_{2}(\bar{F})\right) \cap \mathcal{W}_{w}^{1}$ such that $\left\langle g_{1}, g_{2}\right\rangle=\widetilde{\Gamma}_{w}^{1}$ (see Proposition 3.1). Further, the set $\mathcal{W}_{w}$ is $\operatorname{Gal}(\bar{F} / F)$-stable. Thus

$$
\left(\sigma^{r}\left(g_{1}\right), \sigma^{r}\left(g_{2}\right)\right) \in \mathcal{W}_{w}^{j}(\bar{F})=\left(\mathrm{SL}_{2}(\bar{F}) \times \mathrm{SL}_{2}(\bar{F})\right) \cap \mathcal{W}_{w}^{j} \text { for some } j=j(r) .
$$

Further,

$$
\left[g_{1}, g_{2}\right] \backsim\left(\begin{array}{cc}
\epsilon_{p} & 0 \\
0 & \epsilon_{p}^{-1}
\end{array}\right) \Rightarrow\left[\sigma^{r}\left(g_{1}\right), \sigma^{r}\left(g_{2}\right)\right] \backsim\left(\begin{array}{cc}
\epsilon_{p}^{2^{r}} & 0 \\
0 & \epsilon_{p}^{-2^{r}}
\end{array}\right) .
$$

Then (4.15) and (4.16) imply that for every $j=1, \ldots, \frac{p-1}{2}$ there exists $r$ such that $\left(\sigma^{r}\left(g_{1}\right), \sigma^{r}\left(g_{2}\right)\right) \in \mathcal{W}_{w}^{j}(\bar{F})$. Since $\sigma^{r}$ is an automorphism of $\mathrm{SL}_{2}(\bar{F})$, we get

$$
\widetilde{\Gamma}_{w}^{j}=\left\langle\sigma^{r(j)}\left(g_{1}\right), \sigma^{r(j)}\left(g_{2}\right)\right\rangle \approx \widetilde{\Gamma}_{w}^{1} .
$$

Let us now consider a more complicated example.

Example 4.14. $\mathrm{w}(\mathrm{x}, \mathrm{y})=\left[[\mathrm{x}, \mathrm{y}], \mathrm{x}[\mathrm{x}, \mathrm{y}] \mathrm{x}^{-1}\right]$.

We have $w \in F_{2}^{2}=\left[\left[F_{2}, F_{2}\right],\left[F_{2}, F_{2}\right]\right]$.

We remind that by $w_{0} \in W$ we denote the non-trivial element of the Weyl group. Note that all elements of the form $\dot{w}_{0}=\left(\begin{array}{cc}0 & \alpha \\ -\alpha^{-1} & 0\end{array}\right) \in N_{G}(T)$ whose image in $W$ is $w_{0}$ belong to the same conjugacy class. We denote this class by $C_{\dot{w}_{0}}$ (and often shorten $\dot{w}_{0}$ to $\dot{w}$ ). 
Theorem 4.15. Each of the varieties $\mathcal{T}_{w}$ and $\mathcal{W}_{w}$ has three irreducible components:

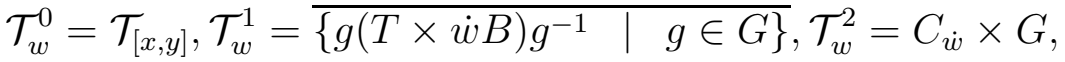

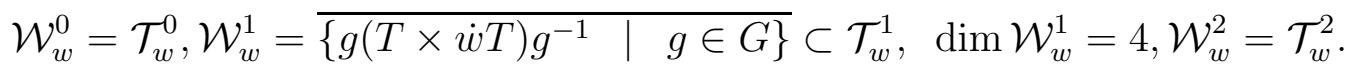

Proof. If $\left(g_{1}, g_{2}\right) \in \mathcal{T}_{[x, y]}$, then $g_{1}$ and $g_{2}$ are in the same Borel subgroup, and therefore $w\left(g_{1}, g_{2}\right)=1$. Hence the 5 -dimensional irreducible variety $\mathcal{T}_{[x, y]}$ coincides with an irreducible component of $\mathcal{T}_{w}$ and $\mathcal{W}_{w}$. Thus we may put

$$
\mathcal{W}_{w}^{0}=\mathcal{T}_{w}^{0}=\mathcal{T}_{[x, y]}
$$

We start with the following lemma.

Lemma 4.16. Let $s \in T, s^{4} \neq 1$, and let $h \in \dot{w} B$. Then $w(s, h)=v \in U^{-}$and

$$
v=1 \Leftrightarrow h=\dot{w} .
$$

Proof. We have $h=\dot{w} u$ for some $\dot{w}$ and some $u \in U$ and

$$
w(s, h)=\left[[s, \dot{w} u], s[s, \dot{w} u] s^{-1}\right] .
$$

Further,

$$
[s, \dot{w} u]=s \dot{w} u s^{-1} u^{-1} \dot{w}^{-1}=\left(s \dot{w} s^{-1}\right) \underbrace{\left(s u s^{-1} u^{-1}\right)}_{=u^{\prime} \in U} \dot{w}^{-1}=\underbrace{\left(s \dot{w} s^{-1} \dot{w}^{-1}\right)}_{=s^{2}} \underbrace{\dot{w} u^{\prime} \dot{w}^{-1}}_{:=v^{\prime} \in U^{-}}=s^{2} v^{\prime}
$$

and $v^{\prime}=1 \Leftrightarrow h=\dot{w}$. Then

$$
s[s, \dot{w} u] s^{-1}=s^{2} \underbrace{\left(s v^{\prime} s^{-1}\right)}_{:=v^{\prime \prime} \in U^{-}}=s^{2} v^{\prime \prime}
$$

and $v^{\prime \prime}=v^{\prime} \Leftrightarrow v^{\prime}=1 \Leftrightarrow h=\dot{w}$. Then

$$
\begin{gathered}
w(s, h)=\left[s^{2} v^{\prime}, s^{2} v^{\prime \prime}\right]=s^{2} v^{\prime} s^{2} \underbrace{v^{\prime \prime} v^{\prime-1}}_{:=v_{1} \in U^{-}} s^{-2} v^{\prime \prime-1} s^{-2}=s^{2} v^{\prime} \underbrace{s^{2} v_{1} s^{-2}}_{:=v_{2} \in U^{-}} v^{\prime \prime-1} s^{-2}= \\
=s^{2} v^{\prime} v_{2} v^{\prime \prime-1} s^{-2}=s^{2} v_{2} \underbrace{v^{\prime} v^{\prime \prime-1}}_{=v_{1}^{-1}} s^{-2}=s^{2} \underbrace{v_{2} v_{1}^{-1}}_{:=v_{3}} s^{-2}=s^{2} v_{3} s^{-2}:=v
\end{gathered}
$$

and $v=1 \Leftrightarrow v_{3}=1 \Leftrightarrow v_{2}=v_{1} \Leftrightarrow v^{\prime \prime}=v^{\prime} \Leftrightarrow h=\dot{w}$.

Lemma 4.16 implies that $\operatorname{tr}(w(s, h))=2$ for every $s \in T$ (if $s^{4}=1$ then $s^{2}= \pm 1$ and $w(s, h)=1$ for every $h \in \dot{w} B)$. Then the set

$$
\left.\overline{\left\{g(T \times \dot{w} B) g^{-1}\right.} \mid g \in G\right\}
$$

is contained in an irreducible component of $\mathcal{T}_{w}$. It is easy to see that

$$
\left.\operatorname{dim} \overline{\left\{g(T \times \dot{w} B) g^{-1}\right.} \mid g \in G\right\}=\operatorname{dim} T+\operatorname{dim} B+\operatorname{dim} G / T=1+2+2=5,
$$

and therefore the set

$$
\left.\overline{\left\{g(T \times \dot{w} B) g^{-1}\right.} \mid g \in G\right\}:=\mathcal{T}_{w}^{1}
$$

is an irreducible component of $\mathcal{T}_{w}$. Further, Lemma 4.16 implies that a general pair $\left(g_{1}, g_{2}\right) \in T \times \dot{w} B$ satisfies the condition $w\left(g_{1}, g_{2}\right)=1$ if and only if $g=\dot{w}$ for some $\dot{w}$. Hence

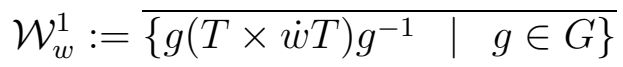


is the only irreducible component of $\mathcal{W}_{w}$ contained in $\mathcal{T}_{w}^{1}$, and

$$
\operatorname{dim} \mathcal{W}_{w}^{1}=\operatorname{dim} T+\operatorname{dim} T+\operatorname{dim} G / T=4 .
$$

Let $\left(g_{1}, g_{2}\right) \in C_{\dot{w}} \times G$. Since $g_{1}^{2}=-1$, we have

$g_{1}\left[g_{1}, g_{2}\right] g_{1}^{-1}=g_{1}\left(g_{1} g_{2} g_{1}^{-1} g_{2}^{-1}\right) g_{1}^{-1}=-g_{2} g_{1}^{-1} g_{2}^{-1} g_{1}^{-1}=-g_{2}\left(-g_{1}\right) g_{2}^{-1} g_{1}^{-1}=\left[g_{2}, g_{1}\right]=\left[g_{1}, g_{2}\right]^{-1}$, and therefore

$$
w\left(g_{1}, g_{2}\right)=\left[\left[g_{1}, g_{2}\right], g_{1}\left[g_{1}, g_{2}\right] g_{1}^{-1}\right]=\left[\left[g_{1}, g_{2}\right],\left[g_{1}, g_{2}\right]^{-1}\right]=1 .
$$

Hence the 5-dimensional variety $C_{\dot{w}} \times G$ coincides with an irreducible component of $\mathcal{W}_{w}$ and also with an irreducible component of $\mathcal{T}_{w}$. Hence we may put

$$
\mathcal{W}_{w}^{2}=\mathcal{T}_{w}^{2}=C_{\dot{w}} \times G .
$$

To prove that neither $\mathcal{T}_{w}$, nor $\mathcal{W}_{w}$ contain additional irreducible components, we need several computational lemmas.

Lemma 4.17. Let $g_{1}=\left(\begin{array}{cc}\lambda & 0 \\ 0 & \lambda^{-1}\end{array}\right), \lambda \neq \pm 1$, and let

$$
g_{2}=\left(\begin{array}{ll}
\alpha & \beta \\
\gamma & \delta
\end{array}\right)
$$

Then

$$
\left[g_{1}, g_{2}\right] \text { is a unipotent element if and only if } g_{2} \in B \cup B^{-}
$$

and

$$
\left\{\begin{array}{l}
{\left[g_{1}, g_{2}\right] \in B \Leftrightarrow \gamma=0 \text { or } \delta=0 \Leftrightarrow g_{2} \in B \text { or } g_{2} \in B \dot{w},} \\
{\left[g_{1}, g_{2}\right] \in B^{-} \Leftrightarrow \beta=0 \text { or } \alpha=0 \Leftrightarrow g_{2} \in B^{-} \text {or } g_{2} \in \dot{w} B}
\end{array} .\right.
$$

Proof. We have (see (4.4))

$$
\left[g_{1}, g_{2}\right]=\left(\begin{array}{cc}
\alpha \delta-\beta \gamma \lambda^{2} & \alpha \beta\left(-1+\lambda^{2}\right) \\
\gamma \delta\left(-1+\lambda^{-2}\right) & \alpha \delta-\beta \gamma \lambda^{-2}
\end{array}\right)
$$

and $\operatorname{tr}\left(\left[g_{1}, g_{2}\right]\right)=2-\beta \gamma\left(\lambda-\lambda^{-1}\right)^{2}$. Therefore,

$$
\operatorname{tr}\left(\left[g_{1}, g_{2}\right]\right)=2 \Leftrightarrow \beta=0 \text { or } \gamma=0 \Leftrightarrow g_{2} \in B \text { or } g_{2} \in B^{-} .
$$

Further,

$$
\left[g_{1}, g_{2}\right] \in B \Leftrightarrow \gamma \delta\left(-1+\lambda^{-2}\right)=0 \Leftrightarrow \gamma=0 \text { or } \delta=0 \Leftrightarrow g_{2} \in B \text { or } g_{2} \in B \dot{w} .
$$

The case $\left[g_{1}, g_{2}\right] \in B^{-}$can be treated by the same arguments.

Lemma 4.18. Let $u$ be a non-trivial unipotent element of $G$, and let $C_{ \pm u}$ be the conjugacy class of $\pm u$. Then $\mathcal{T}_{w}^{j} \neq C_{ \pm u} \times G$. 
Proof. Obviously, we may consider the case $C_{u}$ where $u=\left(\begin{array}{ll}1 & 1 \\ 0 & 1\end{array}\right)$. Take

$$
g=\left(\begin{array}{cc}
i \frac{\sqrt{2}}{2} & -i \frac{\sqrt{2}}{2} \\
-i \sqrt{2} & 0
\end{array}\right)
$$

Then

$$
\begin{gathered}
z=[u, g]=\left(\begin{array}{ll}
1 & 1 \\
0 & 1
\end{array}\right)\left(\begin{array}{cc}
i \frac{\sqrt{2}}{2} & -i \frac{\sqrt{2}}{2} \\
-i \sqrt{2} & 0
\end{array}\right)\left(\begin{array}{cc}
1 & -1 \\
0 & 1
\end{array}\right)\left(\begin{array}{cc}
0 & i \frac{\sqrt{2}}{2} \\
i \sqrt{2} & i \frac{\sqrt{2}}{2}
\end{array}\right)= \\
=\left(\begin{array}{cc}
-i \frac{\sqrt{2}}{2} & -i \frac{\sqrt{2}}{2} \\
-i \sqrt{2} & 0
\end{array}\right)\left(\begin{array}{cc}
-i \sqrt{2} & 0 \\
i \sqrt{2} & i \frac{\sqrt{2}}{2}
\end{array}\right)=\left(\begin{array}{cc}
0 & \frac{1}{2} \\
-2 & 0
\end{array}\right) .
\end{gathered}
$$

Further,

$$
\begin{gathered}
w(u, g)=\left[z, u z u^{-1}\right]=\left(z u z u^{-1}\right)\left(z^{-1} u z^{-1} u^{-1}\right)=\left(z u z u^{-1}\right)\left((-z) u(-z) u^{-1}\right) \\
=\left(z u z u^{-1}\right)^{2}=\left(\left(\begin{array}{cc}
0 & \frac{1}{2} \\
-2 & 0
\end{array}\right)\left(\begin{array}{cc}
1 & 1 \\
0 & 1
\end{array}\right)\left(\begin{array}{cc}
0 & \frac{1}{2} \\
-2 & 0
\end{array}\right)\left(\begin{array}{cc}
1 & -1 \\
0 & 1
\end{array}\right)\right)^{2}= \\
=\left(\left(\begin{array}{cc}
0 & \frac{1}{2} \\
-2 & -2
\end{array}\right)\left(\begin{array}{cc}
0 & \frac{1}{2} \\
-2 & 2
\end{array}\right)\right)^{2}=\left(\begin{array}{cc}
-1 & 1 \\
4 & -5
\end{array}\right)^{2} .
\end{gathered}
$$

The latter matrix is not unipotent because its trace $\neq 2$. Thus we get a contradiction with our assumption.

Lemma 4.19. Let $\mathcal{T}_{w}^{j} \neq \mathcal{T}_{w}^{0}, \mathcal{T}_{w}^{1}, \mathcal{T}_{w}^{2}$ be an irreducible component of $\mathcal{T}_{w}$, and let $\left(g_{1}, g_{2}\right) \in$ $\mathcal{T}_{w}^{j}$ be a general pair. Then $g_{1}$ is a semisimple element of order $\neq 1,2,4,\left[g_{1}, g_{2}\right]$ is a semisimple element, and $\left[g_{1}, g_{2}\right] \neq \pm 1$.

Proof. Suppose that the projection of $\mathcal{T}_{w}^{j} \subset G \times G$ onto the first component is contained in a single conjugacy class $C$ of $G$. Since $\mathcal{T}_{w}^{j}$ is a closed subset invariant under $G$-conjugation and $\operatorname{dim} T_{w}^{j}=5$, we have $\mathcal{T}_{w}^{j}=C \times G$. Lemma 4.18 implies that $C$ is a semisimple class of order $\neq 1,2$. Also, it cannot be of order 4 because this would imply $\mathcal{T}_{w}^{j}=\mathcal{T}_{w}^{1}$. So the order of $g_{1}$ is not equal to $1,2,4$.

Suppose that $\mathcal{T}_{w}^{j} \neq C \times G$.

Let $\operatorname{pr}_{1}: G \times G \rightarrow G$ be the projection onto the first component. Then the set $\operatorname{pr}_{1}\left(\mathcal{T}_{w}^{j}\right)$ is not contained in a single conjugacy class. Since $\overline{\operatorname{tr}\left(\operatorname{pr}_{1}\left(\mathcal{T}_{w}^{j}\right)\right)}$ is an irreducible closed subset of $K$, we have $\overline{\operatorname{tr}\left(\operatorname{pr}_{1}\left(\mathcal{T}_{w}^{j}\right)\right)}=K$, and therefore for a general pair $\left(g_{1}, g_{2}\right) \in \mathcal{T}_{w}^{j}, g_{1}$ is a semisimple element of infinite order.

We may now assume $g_{1}=t \in T$ and $t^{4} \neq 1$. Let $(t, g) \in \mathcal{T}_{w}^{j}$ be a general pair. Suppose $[t, g]$ is a unipotent element. Then $g \in B$ or $g \in B^{-}$by Lemma 4.17. Hence $(t, g) \in T \times B$ or $(t, g) \in T \times B^{-}$, and therefore

$$
(t, g) \in\left\{h(B \times B) h^{-1} \quad \mid h \in G\right\} \subset \mathcal{T}_{[x, y]}^{0} .
$$

This contradicts the assumption $\mathcal{T}_{w}^{j} \neq \mathcal{T}_{w}^{0}$. Thus $\left[g_{1}, g_{2}\right]$ is a semisimple element (if $\left[g_{1}, g_{2}\right]=[t, g]=-u$ where $u \neq 1$ is a unipotent element, then $-u$ and $-t u t^{-1}$ belong to different Borel subgroups by (4.5), (4.6), and therefore $\left(g_{1}, g_{2}\right)=(t, g) \notin \mathcal{T}_{w}$ by Lemma 4.17). 
Suppose now $[t, g]=1$. Then the general pair $(t, g)$ belongs to $T \times T$ and therefore $(t, g) \in \mathcal{W}_{[x, y]}^{0} \subset \mathcal{T}_{w}^{0}$, once again contradicting the assumption $\mathcal{T}_{w}^{j} \neq \mathcal{T}_{w}^{0}$.

Suppose $[t, g]=-1$. We have (see (4.4))

$$
\begin{gathered}
{[t, g]=\left(\begin{array}{cc}
\alpha \delta-\beta \gamma \lambda^{2} & \alpha \beta\left(-1+\lambda^{2}\right) \\
\gamma \delta\left(-1+\lambda^{-2}\right) & \alpha \delta-\beta \gamma \lambda^{-2}
\end{array}\right)=\left(\begin{array}{cc}
-1 & 0 \\
0 & -1
\end{array}\right) \Rightarrow} \\
t= \pm\left(\begin{array}{cc}
i & 0 \\
0 & -i
\end{array}\right), g=\left(\begin{array}{cc}
0 & \mu \\
-\mu^{-1} & 0
\end{array}\right) .
\end{gathered}
$$

This is a contradiction with the choice of $t$.

Lemma 4.20. Let $g \in G$ be a semisimple element of order different from 1,2,4, let $h \in G$, and suppose that $s=[g, h]$ is a semisimple element $\neq \pm 1$. If $s$ and $\mathrm{gsg}^{-1}$ are in the same Borel subgroup, then $s$ and $g$ are also in the same Borel subgroup.

Proof. We may assume $s \in T$ and $s, g s g^{-1} \in B$. If $g \notin B$, then $g=b_{1} \dot{w} b_{2}$ for some $b_{1}, b_{2} \in B$ and

$$
g s g^{-1} \in B \Leftrightarrow b_{1} \dot{w} b_{2} s b_{2}^{-1} \dot{w}^{-1} b_{1}^{-1} \in B \Leftrightarrow \dot{w} b_{2} s b_{2}^{-1} \dot{w}^{-1} \in B \Leftrightarrow b_{2} \in T .
$$

Thus, if $s, g s g^{-1} \in B$ and $g \notin B$ then $g=b \dot{w}$ for some $b \in B$. Then we have

$$
[b \dot{w}, h]=s \in T
$$

for some $b \in B, s \in T$ and $\gamma \in G$. The assumption that $g=b \dot{w}$ is an element of order $\neq 4$ implies that $b \neq 1$. Conjugating both sides of (4.17) with an appropriate element $s^{\prime} \in T$, we can get

$$
\left[\left(\begin{array}{cc}
\rho & 1 \\
-1 & 0
\end{array}\right),\left(\begin{array}{ll}
\alpha & \beta \\
\gamma & \delta
\end{array}\right)\right]=\left(\begin{array}{cc}
\xi & 0 \\
0 & \xi^{-1}
\end{array}\right)
$$

for some $\rho, \alpha, \beta, \gamma, \delta, \xi \in \mathbb{C}$. A straightforward calculation shows that

$$
\left[\left(\begin{array}{cc}
\rho & 1 \\
-1 & 0
\end{array}\right),\left(\begin{array}{ll}
\alpha & \beta \\
\gamma & \delta
\end{array}\right)\right]=\left(\begin{array}{cc}
* & * \\
-\alpha \gamma-\delta \beta+\rho \beta \gamma & *
\end{array}\right)
$$

Then (4.18) and (4.19) imply that $-\alpha \gamma-\delta \beta+\rho \beta \gamma=0$. Since $\alpha \delta-\beta \gamma=1$, we have

$$
\left\{\begin{array} { l } 
{ - \alpha \gamma - \delta \beta + \rho \beta \gamma = 0 } \\
{ \alpha \delta - \beta \gamma = 1 }
\end{array} \Rightarrow \left\{\begin{array}{l}
\gamma=-\frac{\beta}{\alpha^{2}+\beta^{2}-\rho \alpha \beta} \\
\delta=\frac{\alpha-\rho \beta}{\alpha^{2}+\beta^{2}-\rho \alpha \beta}
\end{array} .\right.\right.
$$

(We omit the case $\alpha^{2}+\beta^{2}-\rho \alpha \beta=0$ because in this case we get $\beta=0$ and then $\gamma=0$. But then $h \in T$ is a semismple element and therefore

$$
[b \dot{w}, h]=b \underbrace{\dot{w} h \dot{w}^{-1}}_{=h^{-1}} b^{-1} h^{-1}=b h^{-1} b^{-1} h^{-1}=\underbrace{\left[b, h^{-1}\right]}_{\notin T} h^{-2} \notin T .
$$

But according to our assumption, $[g, h] \in T$.)

We now substitute (4.20) into (4.19) and obtain

$$
\left[\left(\begin{array}{cc}
\rho & 1 \\
-1 & 0
\end{array}\right),\left(\begin{array}{cc}
\alpha & \beta \\
-\frac{\beta}{\alpha^{2}+\beta^{2}-\rho \alpha \beta} & \frac{\alpha-\rho \beta}{\alpha^{2}+\beta^{2}-\rho \alpha \beta}
\end{array}\right)\right]=\left(\begin{array}{cc}
* & \rho\left(1-\left(\alpha^{2}+\beta^{2}-\rho \alpha \beta\right)\right) \\
0 & \alpha^{2}+\beta^{2}-\rho \alpha \beta
\end{array}\right) .
$$


Comparing (4.18) and (4.21), we get $\rho\left(1-\left(\alpha^{2}+\beta^{2}-\rho \alpha \beta\right)\right)=0$. According to our assumption, $\rho \neq 0$. Therefore $\alpha^{2}+\beta^{2}-\rho \alpha \beta=1$, and we get as the commutator in (4.21) a matrix of the form

$$
s=\left(\begin{array}{cc}
\xi & 0 \\
0 & \xi^{-1}
\end{array}\right)=\left(\begin{array}{ll}
* & 0 \\
0 & 1
\end{array}\right) .
$$

Hence $s=1$. This is a contradiction with our assumption. Thus, $s, g \in B$. The lemma is proved.

We now continue the proof of the theorem by showing that $\mathcal{T}_{w}$ has only three irreducible components $\mathcal{T}_{w}^{0}, \mathcal{T}_{w}^{1}, \mathcal{T}_{w}^{2}$. Indeed, assume that there is a component $\mathcal{T}_{w}^{3}$. Lemma 4.19 implies that a general pair $\left(g_{1}, g_{2}\right) \in \mathcal{T}_{w}^{3}$ satisfies the following conditions: $g_{1},\left[g_{1}, g_{2}\right]$ are semisimple elements where the order of $g_{1}$ is not 1,2 , or 4 and $\left[g_{1}, g_{2}\right] \neq \pm 1$. Further, since $\operatorname{tr}\left(w\left(g_{1}, g_{2}\right)\right)=2$, the element

$$
w\left(g_{1}, g_{2}\right)=\left[\left[g_{1}, g_{2}\right], g_{1}\left[g_{1}, g_{2}\right] g_{1}^{-1}\right]
$$

is unipotent, and the elements $\left[g_{1}, g_{2}\right], g_{1}\left[g_{1}, g_{2}\right] g_{1}^{-1}$ belong to the same Borel subgroup according to Lemma 4.17. Then Lemma 4.20 implies that $\left[g_{1}, g_{2}\right], g_{1}$ also belong to the same Borel subgroup. We may assume $g_{1} \in T$ and $\left[g_{1}, g_{2}\right] \in B$. Then applying Lemma 4.17 once again, we get $g_{2} \in B$ or $g_{2} \in B \dot{w}$. Thus $\left(g_{1}, g_{2}\right) \in \mathcal{T}_{w}^{0}$ or $\left(g_{1}, g_{2}\right) \in \mathcal{T}_{w}^{1}$ (note that the pair $(t, b \dot{w})$, where $t \in T$ and $b \in B$, belongs to $\mathcal{T}_{w}^{1}$ because $\left.\dot{w}(t, b \dot{w}) \dot{w}^{-1}=\left(t^{-1}, \dot{w} b\right)\right)$. This contradicts the assumption that $\left(g_{1}, g_{2}\right)$ is a general pair in $\mathcal{T}_{w}^{3} \neq \mathcal{T}_{w}^{0}, \mathcal{T}_{w}^{1}$.

Since $\mathcal{W}_{w}^{0}=\mathcal{T}_{w}^{0}, \mathcal{W}_{w}^{2}=\mathcal{T}_{w}^{2}$ and $\mathcal{W}_{w}^{1}$ is the only irreducible component of $\mathcal{W}_{w}$ contained in $\mathcal{T}_{w}^{1}$, we conclude that $\mathcal{W}_{w}$ also contains only three irreducible components. The theorem is proved.

Corollary 4.21. Let $w$ be a word appearing in any of Examples 4.1. 4.3, 4.7, 4.10, 4.14. Then the induced map $\widetilde{w}: \operatorname{PSL}(2, \mathbb{C})^{2} \rightarrow \operatorname{PSL}(2, \mathbb{C})$ is surjective.

Proof. By Corollary 2.7, the image of $\widetilde{w}$ contains all semisimple elements of $\operatorname{PSL}(2, \mathbb{C})$. The computations of this section provide a 4-dimensional component of $\mathcal{W}_{w}$ for each $w$, hence guarantee that all unipotent elements lie in the image of $\widetilde{w}$. As every element of $\operatorname{PSL}(2, \mathbb{C})$ is either semisimple, or unipotent, we are done.

Remark 4.22. Note that to prove the corollary, we do not need the complete lists of the irreducible components of the varieties $\mathcal{W}_{w}$ (whose computation may be technically involved enough, as in Example 4.14). It suffices to find a 4-dimensional component, which is much easier.

Remark 4.23. Towards computer-aided search of eventual examples of words $w$ such that $G=\mathrm{SL}(2, \mathbb{C})$ contains unipotents lying outside the image of $\widetilde{w}$, it would be important to make the calculation of irreducible components faster. Towards this end, it makes sense to replace the representation variety $\mathcal{W}_{w}$ with the character variety $\mathcal{X}_{w}=\mathcal{W}_{w} / / G$.

Remark 4.24. An approach to proving that all unipotent elements lie in the image of a word map $\widetilde{w}$ where $w \notin F_{m}^{2}$ for every simple algebraic group over fields of characteristic zero is based on the Magnus embedding theorem, combined with the Jacobson-Morozov theorem, see [BZ]. 
Remark 4.25. It is tempting to use representation varieties of associative and Lie algebras (see, e.g., [Na, Remark 1.5]) to study the images of polynomial maps on such algebras, with an eye towards solving some problems raised in [KBMR], [BGKP], [KBKP].

Acknowledgements. The research of the first author was supported by the Ministry of Education and Science of the Russian Federation and RFBR grant 14-01-00820. The research of the second and third authors was supported by ISF grants 1207/12, 1623/16 and the Emmy Noether Research Institute for Mathematics.

We thank M. Borovoi for providing the reference [GOV]. We are grateful to the referee for careful reading and useful remarks.

\section{REFERENCES}

[BGK] T. Bandman, S. Garion, B. Kunyavskii, Equations in simple matrix groups: algebra, geometry, arithmetic, dynamics, Cent. Eur. J. Math. 12 (2014), 175-211.

[BGKP] T. Bandman, N. Gordeev, B. Kunyavskii, E. Plotkin, Equations in simple Lie algebras, J. Algebra 355 (2012), 67-79.

[BZ] T. Bandman, Yu. G. Zarhin, Surjectivity of certain word maps on $P S L(2, \mathbb{C})$ and $S L(2, \mathbb{C})$, European J. Math 2 (2016), 614-643.

[Bo1] A. Borel, On free subgroups of semisimple groups, Enseign. Math. 29 (1983), 151-164.

[Bo2] A. Borel, Linear Algebraic Groups, 2nd ed., Springer-Verlag, New York, 1991.

[Bou] N. Bourbaki, Groupes et algèbres de Lie, chap. IV-VI, Hermann, Paris, 1968.

[Ch1] P. Chatterjee, On the surjectivity of the power maps of algebraic groups in characteristic zero, Math. Res. Lett. 9 (2002), 741-756.

[Ch2] P. Chatterjee, On the surjectivity of the power maps of semisimple algebraic groups, Math. Res. Lett. 10 (2003), 625-633.

[ET] A. Elkasapy, A. Thom, About Goto's method showing surjectivity of word maps, Indiana Univ. Math. J. 63 (2014), 1553-1565.

[FT] A. Fel'shtyn, E. Troitsky, Aspects of the property $R_{\infty}$, J. Group Theory 18 (2015), $1021-1034$.

[GOV] V. V. Gorbatsevich, A. L. Onishchik, E. B. Vinberg, Structure of Lie groups and Lie algebras, "Current Problems in Mathematics. Fundamental directions", vol. 41, "Lie Groups and Lie Algebras. III", VINITI, Moscow, 1990; English transl. in Encyclopaedia Math. Sci., vol. 41, Springer-Verlag, Berlin et al., 1994.

[G1] N. Gordeev, Products of conjugacy classes in algebraic groups, J. Algebra 173 (1995), 715-744.

[G2] N. Gordeev, Freedom in conjugacy classes of simple algebraic groups and identities with constants, Algebra i Analiz 9 (1997), no. 4, 63-78; English transl. in St. Petersburg Math. J. 9 (1998), 709-723.

[G3] N. Gordeev, On Engel words on simple algebraic groups, J. Algebra 425 (2015), 215-244.

[GKP] N. L. Gordeev, B. Ė. Kunyavskiǔ, E. B. Plotkin, Word maps amd word maps with constants of simple algebraic groups, Dokl. Akad. Nauk 471 (2016), no. 2, 136-138; English transl. in Dokl. Math. 94 (2016), 632-634.

[KBKP] A. Kanel-Belov, B. Kunyavskiı̆, E. Plotkin, Word equations in simple groups and polynomial equations in simple algebras, Vestnik St. Petersburg Univ. Math. 46 (2013), no. 1, 3-13.

[KBMR] A. Kanel-Belov, S. Malev, L. Rowen, The images of non-commutative polynomials evaluated on $2 \times 2$ matrices, Proc. Amer. Math. Soc. 140 (2012), 465-478.

[KT] A. Klyachko, A. Thom, New topological methods to solve equations over groups, arXiv:1509.01376.

[Le] M. Levy, Word values in finite groups, Ph.D. thesis, Imperial College, London, 2014.

[LM] A. Lubotzky, A. R. Magid, Varieties of representations of finitely generated groups, Mem. Amer. Math. Soc. 58 (1985), no. 336. 
[Na] K. Nakamoto, Representation varieties and character varieties, Publ. Res. Inst. Math. Sci. 36 (2000), 159-189.

[NS1] N. Nikolov, D. Segal, On finitely generated profinite groups, I. Strong completeness and uniform bounds, Ann. Math. 165 (2007), 171-238; II. Products in quasisimple groups, ibid., 239-273.

[NS2] N. Nikolov, D. Segal, Generators and commutators in finite groups; abstract quotients of compact groups, Invent. Math. 190 (2012), 513-602.

[Pl] V. P. Platonov, Rings and varieties of representations of finitely generated groups, "Problems in algebra", no. 4, Universitetskoe, Minsk, 1989, pp. 36-40. (Russian)

[PBK] V. P. Platonov, V. V. Benyash-Krivets, Rings of characters of $n$-dimensional representations of finitely generated groups, Dokl. Akad. Nauk SSSR 289 (1986), no. 2, 293-297. (Russian)

[RBKC] A. S. Rapinchuk, V. V. Benyash-Krivetz, V. I. Chernousov, Representation varieties of the fundamental groups of compact orientable surfaces, Israel J. Math. 93 (1996), 29-71.

[Ri] R. W. Richardson, Commuting varieties of semisimple Lie algebras and algebraic groups, Compositio Math. 38 (1979), 311-327.

[Se] D. Segal, Words: notes on verbal width in groups, London Math. Soc. Lecture Note Ser., vol. 361, Cambridge Univ. Press, Cambridge, 2009.

[Shaf] I. R. Shafarevich, Basic Algebraic Geometry, 1, 3rd ed., Springer, Heidelberg, 2013.

[Shal] A. Shalev, Some results and problems in the theory of word maps, in: "Erdös Centennial" (L. Lovász, I. Ruzsa, V. T. Sós, D. Palvolgyi, Eds.), Bolyai Soc. Math. Studies, vol. 25, Springer, 2013, pp. 611-649.

[Si] A. S. Sikora, Character varieties, Trans. Amer. Math. Soc. 364 (2012), 5173-5208.

[Sp] T. A. Springer, Twisted conjugacy in simply connected groups, Transform. Groups 11 (2006), $539-545$.

[SS] T. A. Springer, R. Steinberg, Conjugacy classes, "Seminar on Algebraic Groups and Related Finite Groups", Lecture Notes Math., vol. 131, Springer, Berlin, 1970, pp. 167-266.

[Stei] R. Steinberg, On power maps in algebraic groups, Math. Res. Lett. 10 (2003), 621-624.

[Ste1] A. Stepanov, Free product subgroups between Chevalley groups $G(\Phi, F)$ and $G(\Phi, F[t])$, J. Algebra 324 (2010), 1549-1557.

[Ste2] A. Stepanov, Subring subgroups in Chevalley groups with doubly laced root systems, J. Algebra $362(2012), 12-29$.

Gordeev: Department of Mathematics, Herzen State Pedagogical University, 48 Moika Embankment, 191186, St.Petersburg, RUSSIA

E-mail address: nickgordeev@mail.ru

Kunyavskǐ̌: Department of Mathematics, Bar-Ilan University, 5290002 Ramat Gan, ISRAEL

E-mail address: kunyav@macs.biu.ac.il

Plotkin: Department of Mathematics, Bar-Ilan University, 5290002 Ramat Gan, isRael

E-mail address: plotkin@macs.biu.ac.il 